\title{
An efficient model of drillstring dynamics
}

\author{
T. Butlin *, R. S. Langley \\ Cambridge University Engineering Department, Trumpington Street, Cambridge, \\ CB2 1PZ, England
}

\section{Highlights}

- Two efficient models of drillstring dynamics are presented;

- Both models exploit an assumption of spatially localised nonlinearities;

- The models allow for bending and torsional vibration and are in good agreement;

- A test system is shown that exhibits stick-slip; forward whirl and backward whirl;

- Parametric studies reveal conditions of high amplitude vibration.

\begin{abstract}
High amplitude vibration regimes can cause significant damage to oilwell drillstrings: torsional stick-slip oscillation, forward whirl and backward whirl are each associated with different kinds of damage. There is a need for models of drillstring dynamics that can predict this variety of phenomena that are: efficient enough to carry out parametric studies; simple enough to provide insight into the underlying physics, and which retain sufficient detail to correlate to real drillstrings. The modelling strategy presented in this paper attempts to balance these requirements. It includes the dynamics of the full length of the drillstring over a wide bandwidth but assumes that the main nonlinear effects are due to spatially localised regions of strong nonlinearity, for example at the drillbit cutting interface and at stabilisers where the borehole wall clearance is smallest. The equations of motion can be formed in terms of this reduced set of degrees of freedom, coupled to the nonlinear contact laws and solved by time-domain integration. Two implementations of this approach are presented, using (1) digital filters and (2) a Finite Element model to describe the linear dynamics. Choosing a sampling period that is less than the group delay between nonlinear degrees of freedom results in a decoupled set of equations that can be solved very efficiently. Several cases are presented which demonstrate a variety of phenomena, including stick-slip oscillation; forward whirl and backward whirl. Parametric studies are shown which reveal the conditions which lead to high amplitude vibration regimes, and an analytic regime boundary is derived for torsional stick-slip oscillation. The digital filter and Finite Element models are shown to be in good agreement and are similarly computationally efficient. The digital filter approach has the advantage of more intuitive interpretation, while the Finite Element model is more readily implemented using existing software packages.
\end{abstract}


Key words: drillstring dynamics, friction, cutting, impact, nonlinear vibration, local nonlinearity, digital filters, finite element, whirl, stick-slip

\section{Introduction}

Vibration problems represent a significant cost to the oilwell drilling industry due to a wide range of problematic phenomena that can occur [1]. Torsional vibration can cause fatigue, damage to the drillbit if the direction of rotation periodically reverses, or even unthreading of the drillpipe segments in extreme cases. Lateral vibration can lead to high amplitude vibration regimes such as whirl (forward or backward) or impacts with the borehole wall. Forward whirl damage is often characterised by localised drillpipe wear (one face of the drillpipe remains in sliding contact with the borehole wall), while backward whirl often occurs at many times the rotation frequency and can lead to fatigue damage. Lateral impact events (transient or sustained) can damage onboard equipment and unwanted axial vibration is most commonly associated with damage to the drillbit.

There is a clear need for predictive models of drillstring vibration that can capture this wide range of phenomena. Ideally, a theoretical model should: realistically include the key physical effects that affect the system behaviour; provide clear insight into the effect of essential parameters; and account for uncertainties. These requirements are in conflict: a high level of model fidelity is usually at the expense of efficiency and clarity.

A variety of approaches have been taken to modelling drillstring vibration: from highly idealised lumped parameter models to high resolution finite element models. Lumped parameter models tend to be difficult to correlate with real systems but are computationally efficient (e.g. Richard et al. [2,3], Kovalyshen et al. [4], and Depouhon and Detournay [5]). On the other hand detailed models with many degrees of freedom may be more realistic, but are computationally expensive and do not necessarily provide clear insight into the effect of parameters on system behaviour (e.g. Khulief et al. [6], Christoforou and Yigit [7], Germay et al. [8], Lui et al. [9]). The literature also reveals a variety of approaches taken to modelling interfaces between the drillstring and the borehole, with particular focus on the cutting interface at the drillbit. The most common approach is to assume that cutting torque is proportional

\footnotetext{
* Corresponding author (tel: +44 1223 748522)

Email addresses: TB267@cam.ac.uk (T. Butlin), RSL21@cam.ac.uk (R. S. Langley).
} 
to weight-on-bit (WOB), with a friction coefficient that decreases with increasing rotation speed (e.g. $[10,11])$. There also exists a body of literature that considers regenerative effects where the surface profile from each cutter provides the input to the next cutter (e.g. $[5,12])$. These effects can be described using delay-differential equations and have been extensively studied in the machine-tool literature (e.g. $[13,14])$. There are many other effects that could be included in a drillstring model, such as fluid-structure interaction, the effect of free particles at frictional and cutting interfaces, well-path tortuosity, mud-pump excitation, and buckling. It is clear that there are strategic choices necessary when developing a model.

This paper describes a modelling approach that steers an intermediate path in terms of complexity. The goal is for a model that includes the essential features of the system dynamics necessary for realistic predictions, while retaining efficiency and clarity. The approach taken is to assume that the most significant nonlinearities occur at spatially localised regions of the structure (e.g. the drillbit cutting interface or 'stabilisers' where the drillstring radius is larger). The equations of motion can be formed in terms of this reduced set of degrees of freedom, coupled to the nonlinear contact laws and solved by time-domain integration. This style of approach was first developed by McIntyre and Woodhouse [15] in the context of modelling frictional interactions for bowed-string instruments and in parallel by Clough and Wilson [16]. The constitutive laws for the nonlinear interfaces have been assumed to be as simple as possible, but still demonstrate the main field-observed phenomena, such as stick-slip, forward whirl or backward whirl.

Two different implementations are described and compared: (1) using digital filters to describe the linear parts of the system; and (2) using a finite element model reduced to the 'nonlinear' degrees of freedom. Section 2 describes the digital filter implementation, which is based on McIntyre and Woodhouse [15] in modelling the vibration of bowed-string instruments. Here we extend the method to allow for an arbitrary number of localised nonlinearities. Section 3 presents the finite element formulation which follows the reduction scheme of Clough and Wilson [16] and results in a similarly efficient computational scheme to the digital filter approach. Although there is no particular computational advantage in using digital filters, they provide greater physical insight into the underlying dynamics. Section 4 presents the interface models used for nonlinear analysis, and in Section 5 the two nonlinear time-domain models are shown to qualitatively exhibit many of the phenomena that are observed in drillstrings, and as such could be a valuable tool for efficient investigation of drillstring dynamics and other applications where nonlinearities can be considered to be spatially localised.

The key contributions of this paper are to: extend the digital filter approach to multiple localised nonlinearities; apply the concept of localised nonlinearities 
and associated model reduction to drillstrings (usually model reduction limits the number of underlying linear modes included, whereas the assumption of local nonlinearity is not based on restricting the simulation bandwidth); and to highlight the similarity between the digital filter and finite element approaches when this reduction is implemented.

\section{Digital filter model}

\subsection{Summary}

The overall strategy is to identify the nonlinear degrees of freedom, then describe the linear system in terms of a matrix of impulse responses $\mathbf{A}(\mathbf{t})$ that relate those degrees of freedom. In this paper the matrix $\mathbf{A}(\mathbf{t})$ is determined using periodic structure theory to describe a chain of drillpipe sections that are threaded together. This allows the detailed geometry of the full length of a drillstring to be included, capturing the detailed dispersion relationships for both torsion and bending waves. For a more general system, the impulse response matrix could be estimated by measurement or from a finite-element model. The structure can be divided into an arbitrary number of subsections at the points where nonlinearities are known (or suspected) to occur.

The response vector $\mathbf{U}(\mathbf{t})$ is given by convolving the impulse response matrix $\mathbf{A}$ with the corresponding external input vector $\mathbf{E}(\mathbf{t})$, i.e. $\mathbf{U}=\mathbf{A} * \mathbf{E}$. The convolution can be discretised and split into past and current states so that for the $k$ th time sample $\mathbf{U}(k)=\mathbf{A}(0) \mathbf{E}(k) / f_{s}+\mathbf{U}_{h}$, where $f_{s}$ is the sampling frequency and $\mathbf{U}_{h}$ is the remainder of the convolution that depends only on past states (subscript $h$ for 'history' terms). By choosing the sampling period $1 / f_{s}$ to be less than the group delay between the chosen degrees of freedom the matrix $\mathbf{A}(0)$ becomes diagonal. Therefore, the resulting equation is a set of uncoupled linear load lines that can be solved together with the nonlinear law at each location independently without any numerical problem in handling discontinuous impact or friction laws.

The following sections describe the method more fully for torsion and bending vibration. Axial vibration could readily be included within the framework, but in the interests of brevity fall beyond the scope of the present paper.

Figure 1 illustrates a single pipe and joint section and provides a definition of variables: length $L_{1,2}$, shear modulus $G$, polar moment of area of the crosssection $J_{1,2}$, density $\rho$, Young's modulus $E$, second moment of area $I_{1,2}$ and cross-sectional area $A_{1,2}$ (subscripts $\{1,2\}$ denote pipe and joint respectively). Note that material properties for the pipe and joint are assumed to be the 
same (i.e. $G_{1}=G_{2}=G, \rho_{1}=\rho_{2}=\rho$, and $E_{1}=E_{2}=E$ ). The displacements $\theta, x$, and $y$ are for each end of the $n$th section (indicated by the subscripts $n$ and $n+1)$, and are functions of axial distance from the surface $\xi$ and time $t$.

\subsection{Torsional vibration}

\subsubsection{Linear model}

The equation of motion for the torsional dynamics of a pipe of uniform crosssection can be written:

$$
G J_{1} \theta^{\prime \prime}-c_{t} \dot{\theta}-\rho J_{1} \ddot{\theta}=0 .
$$

where $\theta(\xi, t)$ is the angular displacement of the drillstring, $\theta^{\prime}=\partial \theta / \partial \xi, \dot{\theta}=$ $\partial \theta / \partial t$, and $c_{t}$ is a torsional viscous damping term that accounts for distributed energy loss in the waveguide in a deliberately simplified fashion.

Letting $\theta=\bar{\theta} e^{i \omega t}$ and rewriting in first-order form for angular velocity gives:

$$
\left[\begin{array}{c}
\overline{\dot{\theta}}^{\prime} \\
G J_{1} \bar{\theta}^{\prime \prime}
\end{array}\right]=\left[\begin{array}{cc}
0 & \frac{i \omega}{G J_{1}} \\
c_{t}+i \omega \rho J_{1} & 0
\end{array}\right]\left[\begin{array}{c}
\overline{\dot{\theta}} \\
G J_{1} \bar{\theta}^{\prime}
\end{array}\right]
$$

The choice of states $\dot{\theta}$ and $G J \theta^{\prime}$ is convenient as Equation 2 relates angular velocity to torque, which allows straightforward coupling to cutting models defined in terms of these variables. To simplify notation let $T=G J_{1} \theta^{\prime}$ and define:

$$
\boldsymbol{\Gamma}_{1}^{(t)}=\left[\begin{array}{cc}
0 & \frac{i \omega}{G J_{1}} \\
c_{t}+i \omega \rho J_{1} & 0
\end{array}\right]
$$

The torsional degrees of freedom at each end of the $n$th pipe and joint section of drillpipe are described by a transfer matrix $\mathbf{M}_{n}^{(t)}$ :

$$
\left[\begin{array}{l}
\bar{\theta}_{n+1} \\
\bar{T}_{n+1}
\end{array}\right]=\mathbf{M}_{n}^{(t)}\left[\begin{array}{l}
\bar{\theta}_{n} \\
\bar{T}_{n}
\end{array}\right]
$$

where $\mathbf{M}_{n}^{(t)}=e^{\Gamma_{2}^{(t)} L_{2}} e^{\boldsymbol{\Gamma}_{1}^{(t)} L_{1}}$. Therefore the states at each end of $\mathrm{N}$ sections of drillpipe are related by:

$$
\left[\begin{array}{l}
\overline{\dot{\theta}}_{N} \\
\bar{T}_{N}
\end{array}\right]=\prod_{n=0}^{N-1} \mathbf{M}_{n}^{(t)}\left[\begin{array}{c}
\overline{\dot{\theta}}_{0} \\
\bar{T}_{0}
\end{array}\right]
$$


The pair of eigenvalues and eigenvectors of $\mathbf{M}_{n}^{(t)}$ correspond to the forward and reverse travelling waves along the periodic pipe. The magnitude of the eigenvalues are a reciprocal pair: $R$ and $1 / R[17]$. Without loss of generality we choose to define $R \geq 1$ so that $R$ also corresponds to the magnitude of the largest eigenvalue of $\mathbf{M}_{n}^{(t)}$. For nonzero damping $R \neq 1$, so the reverse travelling wave solution appears to grow exponentially with increasing $n$. For very long drillpipes, the product of $\mathbf{M}_{n}^{(t)}$ becomes ill-conditioned, which can be avoided by suitable scaling.

The matrix $\mathbf{M}_{n}^{(t)}$ can be written in terms of its eigenvalues and eigenvectors: $\mathbf{M}_{n}^{(t)}=\boldsymbol{\Phi} \boldsymbol{\Lambda} \boldsymbol{\Psi}^{T}$, where $\boldsymbol{\Lambda}$ is a diagonal matrix of eigenvalues and $\boldsymbol{\Phi}$ and $\boldsymbol{\Psi}$ are the right and left eigenvector matrices respectively. A given solution at the origin $(n=0)$ can be expressed as a linear combination of these eigenvectors:

$$
\left[\begin{array}{c}
\overline{\dot{\theta}}_{0} \\
\bar{T}_{0}
\end{array}\right]=\mathbf{\Phi} \Lambda^{0} \mathbf{a}
$$

where a represents the contribution of each eigenvector (columns of $\boldsymbol{\Phi}$ ). The states $N$ sections away are given by:

$$
\left[\begin{array}{l}
\overline{\dot{\theta}}_{N} \\
\bar{T}_{N}
\end{array}\right]=\boldsymbol{\Phi} \boldsymbol{\Lambda}^{N} \mathbf{a}
$$

Subdividing $\boldsymbol{\Phi}$ such that $\boldsymbol{\Phi}=\left[\begin{array}{ll}P & Q\end{array}\right]^{T}$ allows angular velocity and torque to be separated:

$$
\left[\begin{array}{c}
\overline{\dot{\theta}}_{0} \\
\bar{\theta}_{N}
\end{array}\right]=\left[\begin{array}{c}
\mathbf{P} \boldsymbol{\Lambda}^{\mathbf{0}} \\
\mathbf{P} \boldsymbol{\Lambda}^{N}
\end{array}\right] \mathbf{a}=\hat{\mathbf{P}} \mathbf{a} \text {, and } \quad\left[\begin{array}{c}
\bar{T}_{0} \\
\bar{T}_{N}
\end{array}\right]=\left[\begin{array}{c}
\mathbf{Q} \boldsymbol{\Lambda}^{\mathbf{0}} \\
\mathbf{Q} \boldsymbol{\Lambda}^{\mathbf{N}}
\end{array}\right] \mathbf{a}=\hat{\mathbf{Q}} \mathbf{a}
$$

hence

$$
\left[\begin{array}{c}
\overline{\dot{\theta}}_{0} \\
\overline{\dot{\theta}}_{N}
\end{array}\right]=\hat{\mathbf{P}} \hat{\mathbf{Q}}^{-\mathbf{1}}\left[\begin{array}{c}
\bar{T}_{0} \\
\bar{T}_{N}
\end{array}\right] \text {, or }\left[\begin{array}{c}
\bar{T}_{0} \\
\bar{T}_{N}
\end{array}\right]=\hat{\mathbf{Q}} \hat{\mathbf{P}}^{-1}\left[\begin{array}{l}
\overline{\dot{\theta}}_{0} \\
\overline{\dot{\theta}}_{N}
\end{array}\right]
$$

This rearrangement allows us to choose a scaling matrix $\mathbf{S}$ such that $\hat{\mathbf{P}} \rightarrow$ $\hat{\mathbf{P} S}$ and $\hat{\mathbf{Q}} \rightarrow \hat{\mathbf{Q} S}$. The dynamic stiffness matrix is unchanged as $\hat{\mathbf{Q}} \hat{\mathbf{P}}^{-\mathbf{1}} \rightarrow$ $\hat{\mathbf{Q S S}}^{-\mathbf{1}} \hat{\mathbf{P}}^{-\mathbf{1}}$, so $\mathbf{S}$ can be chosen to eliminate the ill-conditioning. A suitable choice is:

$$
\mathbf{S}=\left[\begin{array}{cc}
R^{-N} & 0 \\
0 & 1
\end{array}\right]
$$

where $R$ is the magnitude of the largest eigenvalue of $\mathbf{M}_{n}^{(t)}$ and is taken to be the first eigenvalue. 
The result is a $2 \times 2$ transfer function matrix $\overline{\mathbf{G}}(i \omega)$ relating angular velocity and torque:

$$
\left[\begin{array}{c}
\overline{\dot{\theta}}_{0} \\
\overline{\dot{\theta}}_{N}
\end{array}\right]=\left[\begin{array}{ll}
\bar{G}_{11} & \bar{G}_{12} \\
\bar{G}_{21} & \bar{G}_{22}
\end{array}\right]\left[\begin{array}{c}
\bar{T}_{0} \\
\bar{T}_{N}
\end{array}\right]
$$

In order to obtain a time domain impulse response the inverse Fourier Transform of the frequency response can be estimated using the inverse Discrete Fourier Transform (this is discussed further in Section 2.4). The discrete-time impulse response gives an approximation to the filter coefficients for a Finite Impulse Response (FIR) digital filter, allowing the response to arbitrary inputs to be computed efficiently. It also allows coupling to localised nonlinearities.

\subsubsection{Coupling to a single nonlinearity}

As a starting point consider a periodic drillstring of $N$ sections, with input angular velocity at the surface $(n=0)$ and in frictional contact at the drillbit $(n=N)$. Continuing in the frequency domain and eliminating $\bar{T}_{0}$ from Equation 11:

$$
\overline{\dot{\theta}}_{N}=\bar{G}_{N N} \bar{T}_{N}+\bar{G}_{N 0} \overline{\dot{\theta}}_{0}
$$

where

$$
\bar{G}_{N N}=\frac{\bar{G}_{11} \bar{G}_{22}-\bar{G}_{12} \bar{G}_{21}}{\bar{G}_{11}} \text {, and } \quad \bar{G}_{N 0}=\frac{\bar{G}_{21}}{\bar{G}_{11}} .
$$

In the time domain this becomes:

$$
\dot{\theta}_{N}=G_{N N} * T_{N}+G_{N 0} * \dot{\theta}_{0}
$$

which can be approximated as a convolution summation, defining $\dot{\theta}_{N}(k) \equiv$ $\dot{\theta}_{N}\left(k / f_{s}\right)$ and where $f_{s}$ is the sampling frequency:

$$
\dot{\theta}_{N}(k)=\sum_{n=0}^{k} G_{N N}(k-n) T_{N}(n) / f_{s}+\sum_{n=0}^{k} G_{N 0}(k-n) \dot{\theta}_{0}(n) / f_{s} .
$$

By taking out the last term in each convolution sum, and noting that $G_{N 0}(0)=0$, this can be written:

$$
\dot{\theta}_{N}(k)=G_{N N}(0) T_{N}(k) / f_{s}+\dot{\theta}_{h}
$$

where $\dot{\theta}_{h}$ represents the 'history' terms of the convolution:

$$
\dot{\theta}_{h} \equiv \sum_{n=0}^{k-1} G_{N N}(k-n) T_{N}(n) / f_{s}+\sum_{n=0}^{k-1} G_{N 0}(k-n) \dot{\theta}_{0}(n) / f_{s} .
$$


Assuming an instantaneous (memoryless) nonlinearity, the torque and angular velocity are related by some friction law:

$$
T_{N}=f\left(\dot{\theta}_{N}\right)
$$

At the $k$ th time-step, Equation 16 and 18 must be solved simultaneously to find the current angular velocity and torque. Equation 16 is simply a linear relation in the $\dot{\theta}_{N}-T_{N}$ plane. The intersection of this with the nonlinear friction-velocity curve gives the current angular velocity and torque explicitly in a method directly analogous to the approach developed to model the bowstring interaction of stringed instruments (McIntyre and Woodhouse [15]).

\subsection{Bending vibration}

\subsubsection{Linear Model}

The equation of motion for the bending dynamics of a pipe of uniform crosssection can be written:

$$
E I_{1} x^{\prime \prime \prime \prime}+C x^{\prime \prime}+\rho A_{1} \ddot{x}+c_{b} \dot{x}=0
$$

where $x(\xi, t)$ is the lateral displacement as a function of distance and time, $\rho$ is the density, $E$ is the Young's modulus, $A_{1}$ is the pipe cross-sectional area, $I_{1}$ is the second moment of area, $c_{b}$ is the viscous damping per unit length, and $C$ is the drillstring pre-compression. Letting $x=\bar{x} e^{i \omega t}$ gives:

$$
E I_{1} x^{\prime \prime \prime \prime}+C \bar{x}^{\prime \prime}-\left(\omega^{2} \rho A_{1}-i \omega c_{b}\right) \bar{x}=0
$$

and the equivalent first-order equation becomes:

$$
\left[\begin{array}{c}
\bar{x}^{\prime} \\
\bar{x}^{\prime \prime} \\
-E I_{1} \bar{x}^{\prime \prime \prime \prime}-C \bar{x}^{\prime \prime} \\
E I_{1} \bar{x}^{\prime \prime \prime}
\end{array}\right]=\left[\begin{array}{cccc}
0 & 1 & 0 & 0 \\
0 & 0 & 0 & \frac{1}{E I_{1}} \\
i \omega c_{b}-\omega^{2} \rho A_{1} & 0 & 0 & 0 \\
0 & -C & -1 & 0
\end{array}\right]\left[\begin{array}{c}
\bar{x} \\
\bar{x}^{\prime} \\
-E I_{1} \bar{x}^{\prime \prime \prime}-C \bar{x}^{\prime} \\
E I_{1} \bar{x}^{\prime \prime}
\end{array}\right] .
$$


To simplify notation let:

$$
\left[\begin{array}{c}
\overline{\mathbf{x}} \\
\hline \overline{\mathbf{F}}_{x}
\end{array}\right]=\left[\begin{array}{c}
\bar{x} \\
\bar{x}^{\prime} \\
\hline-E I_{1} \bar{x}^{\prime \prime \prime}-C \bar{x}^{\prime} \\
E I_{1} \bar{x}^{\prime \prime}
\end{array}\right] \text {, and } \boldsymbol{\Gamma}_{1}^{(b)}=\left[\begin{array}{cccc}
0 & 1 & 0 & 0 \\
0 & 0 & 0 & \frac{1}{E I_{1}} \\
i \omega c_{b}-\omega^{2} \rho A_{1} & 0 & 0 & 0 \\
0 & -C & -1 & 0
\end{array}\right]
$$

where $\overline{\mathbf{x}}$ and $\overline{\mathbf{F}}_{x}$ represent states associated with displacement and force respectively. Note that $\overline{\mathbf{F}}_{x}(1)$ is the shear and $\overline{\mathbf{F}}_{x}(2)$ is the bending moment. This allows Equation 22 to be written more compactly:

$$
\left[\begin{array}{c}
\overline{\mathbf{x}}^{\prime} \\
\overline{\mathbf{F}}_{x}^{\prime}
\end{array}\right]=\boldsymbol{\Gamma}_{1}^{(b)}\left[\begin{array}{c}
\overline{\mathbf{x}} \\
\overline{\mathbf{F}}_{x}
\end{array}\right] .
$$

The states relating to bending for the $n$th section of pipe and joint are governed by a transfer matrix $\mathbf{M}_{n}^{(b)}=e^{\Gamma_{2}^{(b)} L_{2}} e^{\Gamma_{1}^{(b)} L_{1}}$ :

$$
\left[\begin{array}{c}
\overline{\mathbf{x}}_{n+1} \\
\overline{\mathbf{F}}_{x, n+1}
\end{array}\right]=\mathbf{M}_{n}^{(b)}\left[\begin{array}{c}
\overline{\mathbf{x}}_{n} \\
\overline{\mathbf{F}}_{x, n}
\end{array}\right] \text {. }
$$

Note that the desired form for these equations is in terms of displacement, rather than velocity as used for torsion. This is because the normal component of nonlinear contact laws are usually defined in terms of displacement, while torsional friction or cutting laws are defined in terms of velocity. This makes it more straightforward to couple to nonlinearities (as described next in Section 2.3.2).

The eigenvalues of $\mathbf{M}_{n}^{(b)}$ correspond to the four general solutions of the bending equations of motion: a forward and reverse travelling wave and two evanescent solutions of the form $\bar{x}=e^{ \pm \beta \xi}$ where $\beta$ is a constant. Scaling is carried out in the same way as for Section 2.2.1, with $\mathbf{S}$ chosen to be:

$$
\mathbf{S}=\left[\begin{array}{cccc}
R_{1}^{-N} & 0 & 0 & 0 \\
0 & R_{2}^{-N} & 0 & 0 \\
0 & 0 & 1 & 0 \\
0 & 0 & 0 & 1
\end{array}\right]
$$

where $R_{1}$ and $R_{2}$ are the eigenvalues of $\mathbf{M}_{n}^{(b)}$ with largest real parts and assuming that they correspond to the first eigenvector-eigenvalue pairs. 
The result is a $4 \times 4$ transfer function matrix $\mathbf{H}(i \omega)$ relating displacements and rotations $(\overline{\mathbf{x}})$ to shear force and bending moments $(\overline{\mathbf{F}})$ :

$$
\left[\begin{array}{c}
\overline{\mathbf{x}}_{0} \\
\overline{\mathbf{x}}_{N}
\end{array}\right]=\overline{\mathbf{H}}(i \omega)\left[\begin{array}{c}
\overline{\mathbf{F}}_{0} \\
\overline{\mathbf{F}}_{N}
\end{array}\right]
$$

The coefficients for the FIR digital filters can then be computed using the inverse DFT as before. In the time-domain, displacements can be calculated using convolutions of the impulse reponses with externally applied loads:

$$
\left[\begin{array}{c}
\mathbf{x}_{0} \\
\mathbf{x}_{N}
\end{array}\right]=\mathbf{H}(t) *\left[\begin{array}{c}
\mathbf{F}_{0} \\
\mathbf{F}_{N}
\end{array}\right]
$$

\subsubsection{Coupling to nonlinearities}

Coupling these filters in the $x-y$ plane to nonlinearities (see Figure 2) can be achieved in much the same way as for the torsional filters, though there is a subtle difference. A driving point velocity impulse response for a torsional waveguide has an initial delta function equal to the system impedance, so the first coefficient of the torsional velocity filter (multiplied by the sampling period) is equal to the torsional impedance at that point. The bending wave filters are in terms of displacement, and the dispersion relation for EulerBernoulli beams results in the first coefficient of the displacement impulse response being close to zero (in the limit as the sampling frequency tends to infinity, this coefficient equals zero). For the test system studied and for the chosen sampling frequency, the first filter coefficient was found to be approximately $10 \%$ of the maximum value of the driving point impulse responses, therefore this coefficient was not neglected in order to preserve numerical stability when the nonlinear feedback loop is closed. This has an interesting numerical implication: normally it is numerically problematic to model an Euler beam undergoing impact with an infinite-stiffness contact (a rigorous discussion can be found in [18]), however this non-zero (albeit small) first coefficient allows the $k_{c} \rightarrow \infty$ limit to be modelled without difficulty. An alternative viewpoint is that the chosen mapping from continuous to discrete time introduces an effective local compliance in the structure which prevents a singularity in the contact force.

For a simple system with free-free boundary conditions, the downhole displacement at the $k$-th time-step can be written in terms of an externally applied downhole lateral force in the $y$-direction: 


$$
\begin{aligned}
x_{N}(k) & =H_{33}(0) F_{x}(k) / f_{s}+\sum_{n=0}^{k-1} H_{33}(k-n) F_{x}(n) / f_{s} \\
& =H_{33}(0) F_{x}(k) / f_{s}+x_{h}
\end{aligned}
$$

where $x_{h}$ is defined to be the 'history' term representing the convolution with the previous force history.

The general set of equations needed to describe the behaviour of the drillstring with a single nonlinearity at the drillbit is as follows:

$$
\begin{aligned}
x(k) & =H(0) F_{x}(k) / f_{s}+x_{h}(k) \\
y(k) & =H(0) F_{y}(k) / f_{s}+y_{h}(k) \\
\dot{\theta}(k) & =G(0) T(k) / f_{s}+\dot{\theta}_{h} \\
{\left[\begin{array}{c}
F_{N} \\
F_{T}
\end{array}\right] } & =\frac{1}{\sqrt{x^{2}+y^{2}}}\left[\begin{array}{rr}
x & y \\
-y & x
\end{array}\right]\left[\begin{array}{c}
F_{x} \\
F_{y}
\end{array}\right] \\
F_{N} & =F_{R}+\mathbf{F}_{\mathbf{I}} \cdot \mathbf{e}_{\mathbf{r}} \\
F_{T} & =F_{F}+\mathbf{F}_{\mathbf{I}} \cdot \mathbf{e}_{\theta} \\
T & =R_{0} F_{F}+f_{1}(\dot{\theta}, \ldots) \\
\frac{F_{F}}{F_{R}} & =f_{2}(\dot{\theta}, \dot{x}, \dot{y}, \ldots) \\
F_{R} & =f_{3}(x, y, \ldots) .
\end{aligned}
$$

Equations 30 to 32 are the filter equations that represent the linear dynamics (bending in $x$ and $y$ together with torsion). Equation 33 is a coordinate rotation from cartesian force components $\left(F_{x}, F_{y}\right)$ to normal and tangential components $\left(F_{N}, F_{T}\right)$. Equations 34 and 35 relate the total external forces $\left(F_{N}\right.$, $\left.F_{T}\right)$ to the reaction forces $\left(F_{R}, F_{F}\right)$ in polar coordinates assuming an input force vector $\mathbf{F}_{\mathbf{I}}$ applied at the centre of the drillpipe cross-section. Equation 36 gives the torque due to friction for side-contact with the borehole wall, which may also incorporate a nonlinear cutting law, for example to accomodate reamers. Equations 37 and 38 depend on the contact model chosen. The geometry and definition of variables is summarised in Figure 2. External input forces $\mathbf{F}_{\mathbf{I}}$ could for example represent forces due to transient disturbances or noise from frictional or cutting contacts.

\subsection{Computing filter coefficients}

It turns out that computing the filter coefficients by direct application of the inverse Discrete Fourier Transform to the frequency responses results in a number of problems: numerical instability; filter end-effects; and non-preservation 
of the drillstring's static properties. These are a result of mapping from a continuous to a discrete domain: i.e. sampling the continuous frequency response at discrete frequencies. First consider the torsional driving-point admittance, which should be positive-real. The full frequency response of the discrete-time filters does not satisfy this property even though its Discrete Fourier Transform does. This results in numerical instability, which is made apparent by considering the system illustrated in Figure 3. Downhole torque $T_{N}$ is related to downhole angular velocity $\dot{\theta}_{N}$ by friction. This can be represented as a nonlinear gain $K_{n l}$ with linearised worst case occurring during sticking when $K_{n l} \rightarrow-\infty$. The system stability is then determined by the Nyquist stability criterion applied to $K_{n l} \bar{G}_{N N}(i \omega)$. The numerical model will only be stable if the frequency response of $\bar{G}_{N N}(i \omega)$ is positive real, such that the '-1' point is never enclosed by the locus of $K_{n l} \bar{G}_{N N}(i \omega)$ for arbitrarily large $K_{n l}$.

One solution is to apply a low-pass filter to make the high-frequency components very small as it is the high-frequency components of $\bar{G}_{N N}(i \omega)$ that have a negative real part so this has the desired effect on stability. Unfortunately this increases the amplitude of end-effects: wrap-around artefacts from the Discrete Fourier Transform where the end of the filter starts to 'anticipate' the beginning. These in turn can be eliminated by windowing, but this affects the zero-frequency component of the filter (the unit step response should converge to ' 1 ' so that the drillbit angular velocity tends to the surface angular velocity). This can be compensated for by applying a suitable offset. Finally, during a 'sticking' phase of a simulation the downhole angular velocity is zero but, if the surface angular velocity is constant, the downhole torque should ramp up in proportion to the drillstring stiffness. This is equivalent to saying that the ramp response of $G_{N N}(t)$ should settle to the inverse of the effective stiffness of the drillstring. This can also be corrected for by applying a suitable correction function, though this can result in non-physical drift behaviour.

All of these problems can in fact be solved more elegantly. The real and imaginary parts of the frequency response of a causal filter should form a discrete Hilbert transform pair:

$$
\begin{aligned}
\operatorname{real}\left(\bar{G}_{m n}\right) & =\operatorname{hilb}\left\{\operatorname{imag}\left(\bar{G}_{m n}\right)\right\} \\
\operatorname{imag}\left(\bar{G}_{m n}\right) & =-\operatorname{hilb}\left\{\operatorname{real}\left(\bar{G}_{m n}\right)\right\}
\end{aligned}
$$

Computing the imaginary part from the real using this relationship preserves the positive-real property of the continuous frequency response. Taking the real part of the inverse Discrete Fourier Transform gives a new FIR filter, which happens to very closely satisfy each of the properties required above 
without needing artificial compensation:

$$
G_{N}(t)=\operatorname{real}\left\{\operatorname{IDFT}\left[\operatorname{real}\left(\bar{G}_{m n}(i \omega)\right)-i \operatorname{hilb}\left\{\operatorname{real}\left(\bar{G}_{m n}(i \omega)\right)\right\}\right]\right\}
$$

This method does not guarantee to preserve the conditions, but it provides a natural method for converting from the continuous system (which intrinsically satisfies the equivalent properties) to its discrete approximation so these properties are not unduly distorted.

Similar issues arise when computing the filter coefficients for the bending dynamics of the system. The difference is that the frequency response matrix for bending is displacement-based rather than velocity-based. Rather than driving point frequency responses being positive-real as was true for velocity (dissipative only), these driving point transfer functions should always have a negative imaginary part. Again, this is important for numerical stability as external nonlinear forces are a function of displacement creating a feedback loop. The most reliable way to maintain these properties was to first make the frequency response velocity-based by multiplying by $i \omega$, then calculate the real part from the imaginary using Equation 39. The real part of the inverse DFT gives the velocity-based filter, and the cumulative sum brings us back to the displacement-based filter that satisfies the frequency-response criteria.

It is important to choose the sampling frequency and resolution appropriately. The sampling frequency needs to be sufficiently high to capture the bandwidth of interest, but too high and the filters become long and convolution speed limits the overall efficiency. Similarly the frequency resolution should be high enough to reasonably approximate resonant peaks, such that the total time duration of the filter is long enough to capture most of the decay envelope of all modes. A common sampling frequency is required for both torsion and bending filters, so this is set by the higher requirement. Their lengths need not be the same, so the frequency resolutions can be different. For an individual section, the first torsion natural frequency is significantly higher than the first bending mode. These modes indicate the stop-band spacing, so to include two torsion stop-bands, the method found to work well in most situations was to choose the sampling frequency as four times the first torsional mode of an individual section of drillpipe. The frequency resolution was chosen by taking approximately five points to resolve each resonant peak, different for torsion and bending. However, this was not fully automatic and some manual choice sometimes proved necessary. In addition, when high frequency resolutions for bending filter calculations were necessary as well as a high sampling frequency the filter lengths became unmanageable. In this situation the bending filters were calculated over a lower bandwidth, and interpolated in the time-domain to equate sampling frequencies: this did not have an adverse effect on the stability properties discussed above. 


\subsection{Coupling torsion and bending to multiple nonlinearities}

The model so far allows coupling of a linear drillstring to arbitrary nonlinearities at the drill-bit. This is now extended to include a discrete number of nonlinearities at arbitrary locations.

\subsubsection{Torsion}

Dividing the drillstring into $P$ sections, the decoupled torsional frequency responses can be written as a block diagonal matrix:

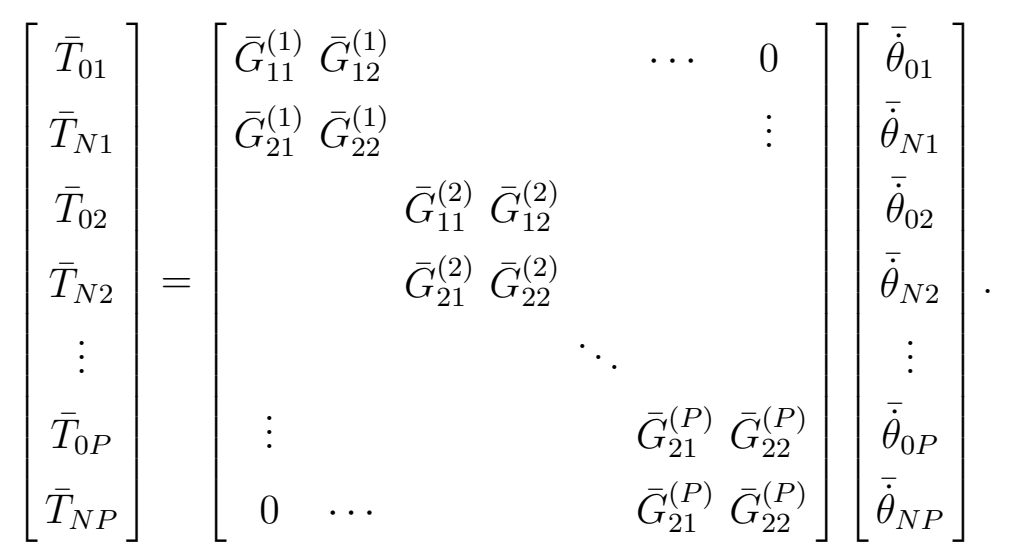

The equilibrium condition between joints $n$ and $n+1$ is:

$$
\bar{T}_{N n}+\bar{T}_{n}^{(e x t)}=\bar{T}_{0(n+1)},
$$

where $T_{n}^{(e x t)}$ is an external torque, and by compatibility:

$$
\overline{\dot{\theta}}_{N n}=\overline{\dot{\theta}}_{0(n+1)} \text {. }
$$

Redefining $\overline{\dot{\theta}}_{j} \equiv \overline{\dot{\theta}}_{N j}$ for compactness, Equation 41 becomes:

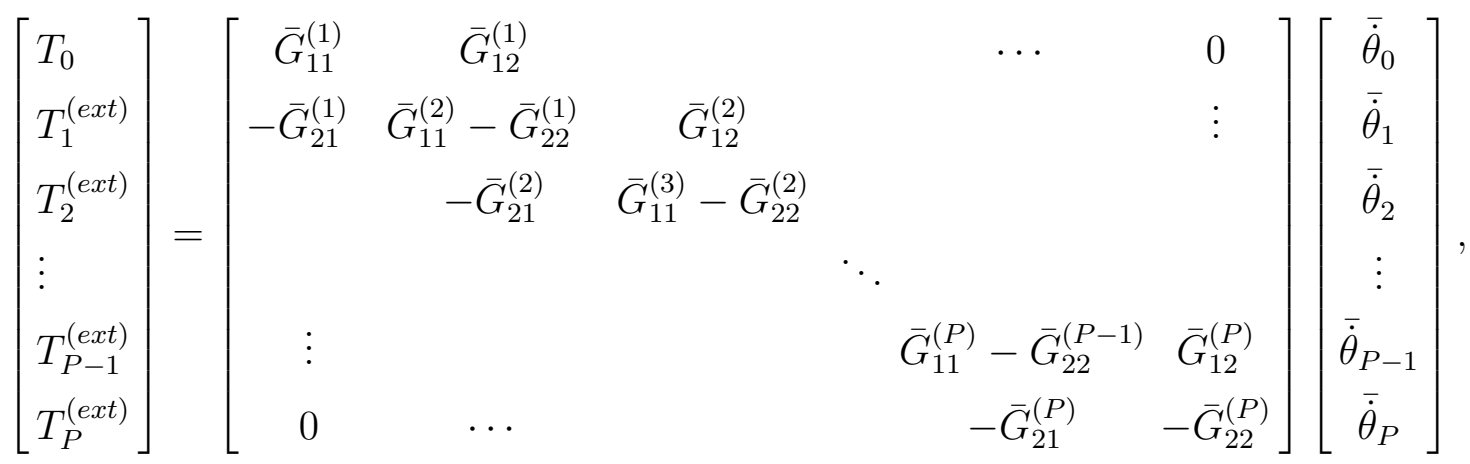


or more compactly, defining the dynamic stiffness matrix for torsion $\overline{\mathbf{K}}^{(t)}$ :

$$
\overline{\mathbf{T}}^{(e x t)}=\overline{\mathbf{K}}^{(t)} \overline{\dot{\boldsymbol{\theta}}}
$$

This can easily be inverted to give output angular velocities in terms of external torques, but this is not exactly what is needed. Usually the surface angular velocity is considered as an input, so only part of the matrix needs inverting. Dropping superscript $(t)$ for clarity and partitioning gives:

$$
\left[\begin{array}{c}
\bar{T}_{0} \\
\overline{\mathbf{T}}^{(e x t)}
\end{array}\right]=\left[\begin{array}{cc}
\bar{K}_{11} & \overline{\mathbf{K}}_{12} \\
\overline{\mathbf{K}}_{21} & \overline{\mathbf{K}}_{22}
\end{array}\right]\left[\begin{array}{c}
\overline{\dot{\theta}}_{0} \\
\overline{\dot{\boldsymbol{\theta}}}
\end{array}\right]
$$

hence outputs can be expressed in terms of inputs:

$$
\left[\begin{array}{c}
\bar{T}_{0} \\
\overline{\boldsymbol{\theta}}
\end{array}\right]=\left[\begin{array}{cc}
\bar{K}_{11}-\overline{\mathbf{K}}_{12} \overline{\mathbf{K}}_{22}^{-1} \overline{\mathbf{K}}_{21} & \overline{\mathbf{K}}_{12} \overline{\mathbf{K}}_{22}^{-1} \\
-\overline{\mathbf{K}}_{22}^{-1} \overline{\mathbf{K}}_{21} & \overline{\mathbf{K}}_{22}^{-1}
\end{array}\right]\left[\begin{array}{c}
\overline{\dot{\theta}}_{0} \\
\overline{\mathbf{T}}^{(e x t)}
\end{array}\right],
$$

and more compactly by defining $\mathbf{D}^{(t)}$ :

$$
\left[\begin{array}{c}
\bar{T}_{0} \\
\overline{\boldsymbol{\theta}}
\end{array}\right]=\overline{\mathbf{D}}^{(t)}\left[\begin{array}{c}
\overline{\dot{\theta}}_{0} \\
\overline{\mathbf{T}}^{(e x t)}
\end{array}\right] \text {. }
$$

Using the Hilbert transform to map to the discrete domain and the inverse DFT (see Equation 40), the time-domain response can be computed using:

$$
\left[\begin{array}{c}
T_{0}(k) \\
\dot{\boldsymbol{\theta}}(k)
\end{array}\right]=\operatorname{diag}\{\mathbf{D}(0)\}\left[\begin{array}{c}
\dot{\theta}_{0}(k) \\
\mathbf{T}^{(e x t)}(k)
\end{array}\right]+\left[\begin{array}{c}
\dot{\theta}_{h} \\
\mathbf{T}_{h}^{(e x t)}
\end{array}\right]
$$

where subscript $h$ represents the convolution of the history of inputs with the digital filters. Note that this is in the same form as Equation 16, but for an arbitrary number of nonlinearities. At a given time-step, the spatially separated nonlinearities do not couple as the first coefficient of all off-diagonal terms in $\mathbf{D}(0)$ is zero. Therefore the coupling of each degree of freedom to nonlinearities at a particular time-step can be solved exactly as in the case of a single nonlinearity.

\subsubsection{Bending}

The same principles can be used to extend the bending model to allow multiple nonlinearities. Again dividing the drillstring into $P$ sections, the decoupled bending frequency responses can be written as a block diagonal matrix: 


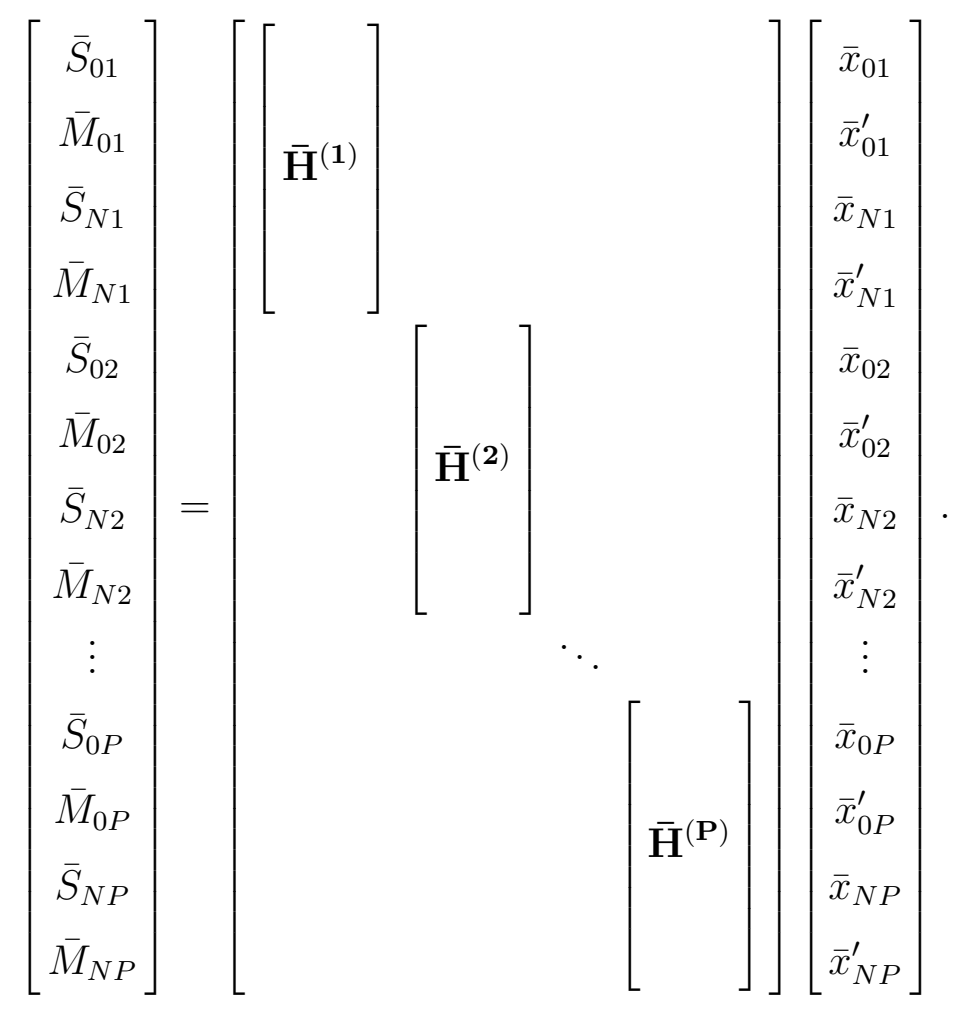

The boundary conditions between joints $n$ and $n+1$ are:

$$
\begin{aligned}
x_{N n} & =x_{0(n+1)} \\
x_{N n}^{\prime} & =x_{0(n+1)}^{\prime} \\
S_{N n}+S_{n}^{(e x t)} & =S_{0(n+1)} \\
M_{N n}+M_{n}^{(e x t)} & =M_{0(n+1)}
\end{aligned}
$$

where $x$ is lateral displacement in the $x$ direction, $S$ is the shear force, $S_{n}^{(e x t)}$ is an external lateral force and $M$ is the bending moment. This allows alternate rows and columns to be combined appropriately. The full matrix equation is somewhat long-winded but the structure of the resulting matrix is: 


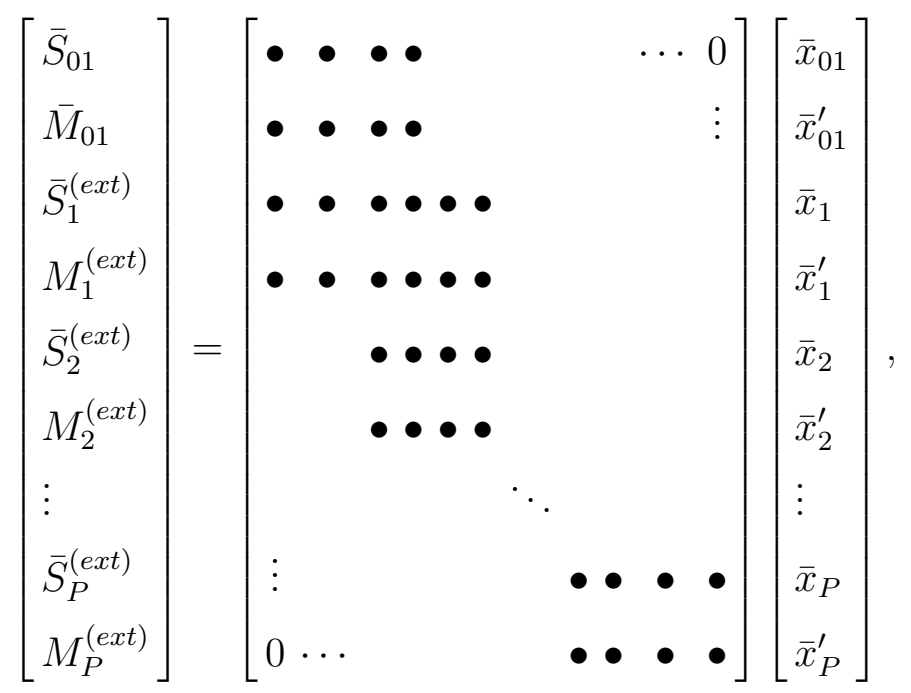

or more compactly, defining the dynamic stiffness matrix for bending $\overline{\mathbf{K}}^{(b)}$ :

$$
\overline{\mathbf{F}}_{x}^{(e x t)}=\overline{\mathbf{K}}^{(b)} \overline{\mathbf{x}}
$$

Defining $\overline{\mathbf{D}}^{(b)}=\overline{\mathbf{K}}^{-1}$, and duplicating the analysis for the $y$ degrees of freedom gives:

$$
\begin{aligned}
& \overline{\mathbf{x}}=\overline{\mathbf{D}}^{(b)} \overline{\mathbf{F}}_{x}^{(e x t)} \\
& \overline{\mathbf{y}}=\overline{\mathbf{D}}^{(b)} \overline{\mathbf{F}}_{y}^{(e x t)}
\end{aligned}
$$

This simplifies further if we only take displacements as outputs (neglecting to calculate $x^{\prime}$ ) and assume that external bending moments are zero on the basis that where impacts occur the borehole wall does not exert a significant moment. This eliminates alternate rows and columns, reducing the number of matrix elements to a quarter of the original.

An FIR filter can be calculated for each term in the resulting frequency response matrix and, as before, can be coupled to each nonlinearity.

\subsection{Combined digital filter model}

The final form of the digital filter equations combining torsion and bending degrees of freedom can be written:

$$
\mathbf{U}(t)=\mathbf{A}(t) * \mathbf{E}(t)
$$


where $\mathbf{U}=\left[\begin{array}{llll}\dot{\theta}_{0} & \mathbf{T}^{(e x t)} & \mathbf{x} & \mathbf{y}\end{array}\right]^{T}, \mathbf{E}=\left[\begin{array}{llll}T_{0} & \dot{\boldsymbol{\theta}} & \mathbf{F}_{x} & \mathbf{F}_{y}\end{array}\right]^{T}$ and $\mathbf{A}$ is the block diagonal matrix of corresponding impulse responses (digital filter coefficients):

$$
\mathbf{A}=\left[\begin{array}{cccc}
D_{11}^{(t)} & \mathbf{D}_{12}^{(t)} & 0 & 0 \\
\mathbf{D}_{21}^{(t)} & \mathbf{D}_{22}^{(t)} & 0 & 0 \\
0 & 0 & \mathbf{D}^{(b)} & 0 \\
0 & 0 & 0 & \mathbf{D}^{(b)}
\end{array}\right]
$$

At a given time step, the nonlinearities are instantaneously decoupled and the response can be written:

$$
\mathbf{U}(k)=\operatorname{diag}\{\mathbf{A}(0)\} \mathbf{E}(k) / f_{s}+\mathbf{U}_{h} .
$$

\section{Finite element model}

A finite element model was implemented in order to verify the digital filter model. The element mass and stiffness matrices for bending and torsion are taken from Rao [19]. Assuming an initially straight drillpipe allows straightforward assembly into consistent global mass and stiffness matrices. The damping matrix was approximated by lumped viscous dashpots (one per element for torsion and two per element for bending) as a simple method for obtaining approximate equivalence with the digital filter model. Converged frequency responses over the bandwidth of interest were obtained using 20 cubic beam elements per segment of drillpipe resulting in approximately $10^{4}$ degrees of freedom for a $1 \mathrm{~km}$ drillstring. The equations of motion can be written in the usual form:

$$
\left[\begin{array}{cc}
\mathbf{M}^{(\mathbf{t})} & \mathbf{0} \\
\mathbf{0} & \mathbf{M}^{(\mathbf{b})}
\end{array}\right]\left[\begin{array}{l}
\ddot{\theta} \\
\ddot{\mathbf{z}}
\end{array}\right]+\left[\begin{array}{cc}
\mathbf{C}^{(\mathbf{t})} & \mathbf{0} \\
\mathbf{0} & \mathbf{C}^{(\mathbf{b})}
\end{array}\right]\left[\begin{array}{c}
\dot{\theta} \\
\dot{\mathbf{z}}
\end{array}\right]+\left[\begin{array}{cc}
\mathbf{K}^{(\mathbf{t})} & \mathbf{0} \\
\mathbf{0} & \mathbf{K}^{(\mathbf{b})}
\end{array}\right]\left[\begin{array}{l}
\boldsymbol{\theta} \\
\mathbf{z}
\end{array}\right]=\left[\begin{array}{c}
\mathbf{T} \\
\overline{\mathbf{F}}
\end{array}\right]
$$

where $\mathbf{M}, \mathbf{C}$ and $\mathbf{K}$ are the assembled global mass, damping and stiffness matrices with superscript (t) for torsion and (b) for bending, $\boldsymbol{\theta}$ is a vector of angular displacements, $\mathbf{z}$ is a combined displacement vector $\mathbf{z}=\left[\begin{array}{ll}\mathbf{x} & \mathbf{y}\end{array}\right]^{T}, \mathbf{T}$ are the external torques and $\mathbf{F}$ are the external lateral forces $\mathbf{F}=\left[\begin{array}{ll}\mathbf{F}_{x} & \mathbf{F}_{y}\end{array}\right]^{T}$. Using the Newmark-Beta integration scheme, the equation at the $k$ th time step can be written in the form (e.g. [20]):

$$
\left[\begin{array}{cc}
\mathbf{K}_{\mathrm{eff}}^{(\mathbf{t})} & \mathbf{0} \\
\mathbf{0} & \mathbf{K}_{\mathrm{eff}}^{(\mathbf{b})}
\end{array}\right]\left[\begin{array}{l}
\boldsymbol{\theta}(k) \\
\mathbf{z}(k)
\end{array}\right]=\left[\begin{array}{c}
\mathbf{T}_{\mathrm{eff}}(k) \\
\mathbf{F}_{\mathrm{eff}}(k)
\end{array}\right]
$$


where $\mathbf{T}_{\text {eff }}(k)$ and $\mathbf{F}_{\text {eff }}(k)$ depend on the external force at the current timestep and the displacement and velocity at the previous time-step. For torsion this can be written:

$$
\mathbf{T}_{\text {eff }}(k)=\mathbf{T}(k)+c_{1} \ddot{\boldsymbol{\theta}}(k-1)+c_{2} \dot{\boldsymbol{\theta}}(k-1)+c_{3} \boldsymbol{\theta}(k-1)
$$

and the coefficients $c_{i}$ for the Newmark-Beta scheme are:

$$
\begin{aligned}
c_{1} & =\left(\frac{1}{2 \beta}-1\right) \mathbf{M}^{(t)}+\frac{1}{f_{s}}\left(\frac{\alpha}{2 \beta}-1\right) \mathbf{C}^{(t)} \\
c_{2} & =\frac{f_{s}}{\beta} \mathbf{M}^{(t)}+\left(\frac{\alpha}{\beta}-1\right) \mathbf{C}^{(t)} \\
c_{3} & =\frac{f_{s}^{2}}{2 \beta} \mathbf{M}^{(t)}+\frac{\alpha f_{s}}{\beta} \mathbf{C}^{(t)}
\end{aligned}
$$

The parameter values initially chosen were: $\alpha=0.5$ and $\beta=0.25$.

The reduction scheme of Clough and Wilson [16] was implemented to reduce the computational cost of numerical integration. Partitioning Equation 63 with respect to the nonlinear interface nodes $(n)$ and internal nodes $(i)$ for torsion gives:

$$
\left[\begin{array}{ll}
\mathbf{K}_{\mathrm{ii}}^{(\mathbf{t})} & \mathbf{K}_{\mathrm{in}}^{(\mathbf{t})} \\
\mathbf{K}_{\mathbf{n i}}^{(\mathbf{t})} & \mathbf{K}_{\mathbf{n n}}^{(\mathbf{t})}
\end{array}\right]\left[\begin{array}{c}
\boldsymbol{\theta}_{\boldsymbol{i}}(k) \\
\boldsymbol{\theta}_{\boldsymbol{n}}(k)
\end{array}\right]=\left[\begin{array}{c}
\mathbf{T}_{\mathbf{i}, \mathbf{e f f}}(k) \\
\mathbf{T}_{\mathbf{n}, \mathbf{e f f}}(k)
\end{array}\right] .
$$

Eliminating $\boldsymbol{\theta}_{\boldsymbol{i}}$ results in:

$$
\boldsymbol{\theta}_{\boldsymbol{n}}(k)=\left(\mathbf{K}_{\mathbf{n n}}-\mathbf{K}_{\mathbf{n i}}^{(\mathbf{t})} \mathbf{K}_{\mathbf{i i}}^{-1} \mathbf{K}_{\mathbf{i n}}\right)^{-1}\left(\mathbf{T}_{\mathbf{n}, \mathbf{e f f}}(k)-\mathbf{K}_{\mathbf{n i}} \mathbf{K}_{\mathbf{i i}}^{-1} \mathbf{T}_{\mathbf{i}, \mathbf{e f f}}(k)\right)
$$

If all non-zero external forces are included within the 'nonlinear' partition then from Equations 63 and 67 this can be written in the form:

$$
\boldsymbol{\theta}_{\boldsymbol{n}}(k)=\mathbf{B T}_{\mathbf{n}}(k)+\boldsymbol{\theta}_{\boldsymbol{h}}
$$

where $\mathbf{B}=\left(\mathbf{K}_{\mathbf{n n}}-\mathbf{K}_{\mathbf{n i}}^{(\mathbf{t})} \mathbf{K}_{\mathbf{i i}}^{-1} \mathbf{K}_{\mathbf{i n}}\right)^{-1}$, and $\boldsymbol{\theta}_{\boldsymbol{h}}$ is a 'history' term that depends only on previous solutions. This is reminiscent of the digital filter formulation of Equation 16, and inspection of $\mathbf{B}$ reveals that it tends towards a diagonal matrix as the sampling frequency $f_{s}$ is increased and the spatial separation of nonlinear nodes $n$ increases. This is expected because there can be no instantaneous coupling between spatially separated degrees of freedom. The same partitioning scheme can be carried out for the bending vibration equations, again assuming that impact with the borehole wall does not exert any moment.

A lagrange multiplier was used to implement the surface angular velocity in- 
put. The velocity state vector and damping matrix were augmented as follows:

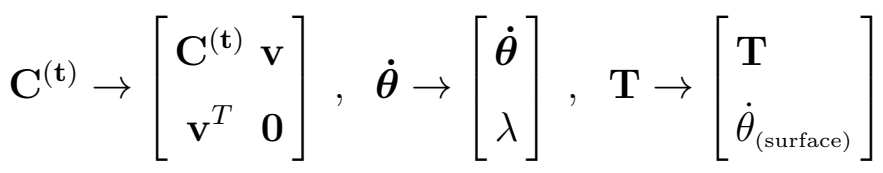

where the first element of $\mathbf{v}$ is one and is zero otherwise.

In summary, at each time step the history term $\boldsymbol{\theta}_{\boldsymbol{h}}(k)$ needs to be evaluated (which involves matrix multiplications that reconstruct all the states and reduce them again) in order to construct the load lines of Equation 68, which can then be solved together with the nonlinear contact laws. Therefore the numerical complexity of the solution scheme is very similar to the digital filter method, and it should come as no surprise that the reduced finite element model is similarly computationally efficient.

\section{Interface Models}

The question remains as to how to appropriately define the nonlinear interface laws when the drillstring makes contact with the borehole wall or at the drillbit. These interactions are the subject of a great deal of research (e.g. [2,21-23]) and a detailed investigation is beyond the scope of the present study. We have chosen a simple model that is in keeping with the premise of developing a lightweight model that is simple but captures the main effects.

\subsection{Borehole wall contact}

The forces acting on the drillstring by the borehole wall are represented by two components: a normal reaction force assumed to follow a Hertzian stiffness law and a tangential frictional force assumed to be independent of sliding velocity.

The normal reaction force $F_{R}$ (as defined in Figure 2) is assumed to be given by:

$$
F_{R}=-k_{c} \delta^{\frac{3}{2}}
$$

where $k_{c}$ is a stiffness coefficient and $\delta$ is the contact depth. It should be noted that both the digital filter model and the Finite Element model work for an infinite contact stiffness and the choice of finite stiffness has been made on physical, rather than numerical, grounds.

The tangential friction force $F_{F}$ is given by:

$$
F_{F}=\mu_{0} F_{R} V_{s} \operatorname{sign}\left\{V_{s}\right\} \quad \forall V_{s} \neq 0
$$


where $V_{s}$ is the relative sliding velocity, i.e. the velocity of the contact point in the $\boldsymbol{e}_{\boldsymbol{\theta}}$ direction (see Figure 2 for the sign convention used). If the sliding velocity is zero, then the position is known by the kinematics of rolling contact and the unknown is the contact force.

\subsection{Cutting laws}

The dynamics of the cutting process perhaps represents the most uncertain nonlinear coupling: the mix of mud-flow with loose cuttings, bit-rock interaction, rock-type and inhomogeneity, coupling to lateral and axial dynamics, regenerative effects and cutter wear all contribute to a highly complex interaction. These effects are not beyond the capability of the modelling strategy, but the cutting model was selected in the spirit of choosing the simplest possible model.

There are several studies investigating the average relationship between the dynamic torque $\left(T_{d}\right)$, weight-on-bit (WOB), and depth of cut per revolution (DOC). Note that the rate-of-penetration (ROP) is intrinsically related to depth of cut per revolution, as $\mathrm{DOC}=\mathrm{ROP} / \mathrm{RPM}$. The approach selected for this study follows Detournay et al. [23], which represents a thorough data-driven investigation that draws out key relationships from experimental drilling tests for two example rock types. The relationships between $T_{d}$, WOB, and DOC were fitted to a piece-wise linear law corresponding to different cutting regimes.

An example is shown in Figure 4(a) for sandstone (dashed, red) and shale (solid, blue) scaled for a $30 \mathrm{~cm}(12 \mathrm{inch}))$ drillbit. This represents a rather large diameter hole consistent with the dimensions of the academic example system described next in Section 5. Figure 4(a) shows torque and (b) shows the depth of cut per revolution, both as a function of weight-on-bit. In the present study, the weight-on-bit is assumed to be constant as axial dynamics have been neglected, and the cutting torque follows the relationship shown in Figure 4(a).

For the following simulations a distinction is made between the dynamic torque limit $T_{d}$ and the static torque limit $T_{s}$ (taken to be $T_{s}=1.2 T_{d}$ ).

\section{Test cases}

A sequence of numerical simulations have been carried out on an idealised drillstring. The aims of this section are to: compare the digital filter approach 
with the finite element model; demonstrate the efficiency achieved by assuming nonlinearities are localised; and qualitatively validate the methodology by simulating commonly observed phenomena.

The idealised geometry used for these initial explorations is summarised in Table 2. The total length is $1 \mathrm{~km}$ and is divided into two sections: $900 \mathrm{~m}$ of periodic drillpipe connected to $100 \mathrm{~m}$ of larger diameter uniform drillcollar representing the Bottom Hole Assembly (BHA). The system parameters have deliberately been chosen to be unrepresentative of industry practice for IP reasons: the results should therefore be interpreted as illustrative. Two scenarios will be considered: (A) with a single nonlinearity at the drillbit (either nonlinear cutting law or borehole wall frictional contact); and (B) with three nonlinearities at discrete stabiliser locations along the BHA (with both cutting and borehole contact included). A sketch is shown in Figure 5 that illustrates these scenarios: the borehole nonlinearity for Scenario (1) is at Site 3 and for Scenario (2) is at Sites 1, 2 and 3. This academic test case exemplifies a wide range of phenomena and provides proof-of-concept for the feasibility of further parametric studies.

For these example simulations, the pre-compression $C$ is chosen to be zero. It's effect has been investigated, but for loads that do not cause buckling it does not significantly affect the qualitative behaviour of the drillstring. Choosing zero compression allows easier comparison with the Finite Element model (for which pre-compression has not been included).

\subsection{Linear Dynamics}

Before carrying out nonlinear simulations, the linear dynamics of the idealised test case will be briefly described. Figure 6(a) shows the reference torsion transfer function from surface angular displacement to downhole angular velocity calculated using periodic structure theory. The filters were calculated over a bandwidth corresponding to two stop-bands of the torsional response: these are clearly visible at approximately $150 \mathrm{~Hz}$ and $300 \mathrm{~Hz}$. There are approximately 100 resonant peaks in-between each stop-band as expected with a 100-segment periodic system. The non-trivial details of the transfer functions arise from the non-uniform periodicity: segments $0-90$ are periodic, while 90 100 is a thicker section of uniform cross-section. The finite element implementation required approximately 2000 elements to provide convergence over the same bandwidth as computed for the digital filters.

Figure 6(b) shows the reference bending driving point transfer function at the bottom end of the drillstring. Damping is significantly higher for bending vibration, and travelling waves in a beam are intrinsically dispersive so there is 
not such an intuitive pattern to the transfer functions. Finite element transfer functions (not shown) over this bandwidth had also converged with 2000 elements.

Figure 7(a) shows the downhole angular velocity response to a $60 \mathrm{rpm}$ step change in surface angular velocity, using both the digital filter and finite element models. It can be seen that the two methods give a very similar response. There is a delay of approximately 0.3 seconds due to the time taken for the torsion wave to travel from surface to bottom and whole round-trip reflections at subsequent 0.6 second intervals are also apparent. The details of the response are more complicated due to significant internal reflections occurring in the thicker $100 \mathrm{~m}$ section at the end of the drillstring. The difference between the two predictions is shown beneath the response: the peak in this difference is due to a small difference in the predicted initial delay. The other differences are due to additional high-frequency signal content in the finite element predictions, most likely a result of differences in numerical damping between the two integration schemes.

Figure 7(b) shows the downhole displacement response to a band-limited unit lateral impulse $(1 \mathrm{Ns})$ at $t=1$ second with duration 0.02 seconds applied at the end of the drillstring. The delay of 1 second before applying the lateral impulse was to ensure that the downhole angular velocity was non-zero at the time of impulse. The difference between the digital filter and finite element predictions is much more obvious: this is partly an artefact of plotting displacement instead of velocity which exaggerates low frequency errors. The difference is most likely because the digital filter integration scheme is inherently first order, while the Newmark-Beta integration scheme of the Finite Element model is second order.

\subsection{Single Contact Behaviour}

\subsubsection{Torsional stick-slip oscillation}

The self-excited torsional vibration of the drillstring has previously been explored in [24] using the digital filter approach. A similar set of simulations is carried out here in order to determine the operating parameter space over which stick-slip oscillation can be sustained and to compare the digital filter predictions with the finite element model. The cutting law is based on drilling through sandstone, using the law described in Section 4.2.

It turns out that the Newmark-Beta integration scheme used for the finite element model is numerically unstable in torsion during sticking, using $\gamma=0.5$ and $\beta=0.25$. It has already been seen that the high frequency behaviour is less damped in the finite element implementation than the digital filter ap- 
proach (e.g. see Figure 7(a)). It may seem surprising that there is any issue of stability here: Newmark-Beta integration with the chosen parameters should be unconditionally stable. The difficulty is that 'sticking' can be viewed as a feedback loop with infinite velocity gain. The issue can be resolved by introducing small numerical damping using $\gamma=0.52$ and $\beta=\frac{1}{4}\left(\frac{1}{2}+\gamma\right)^{2}=0.2601$.

Two example time-domain simulation using the two methods is shown in Figure 8, which will be referred to as case (a) and (b) according to the subfigure labels. It can be seen that both predictions undergo an initial 'sticking' phase, before suddenly slipping. The dynamic friction is assumed to be less than the static friction $\left(T_{s}=1.2 T_{d}\right)$, so the step change in torque sends a travelling wave up the drillstring which is reflected at the surface and travels back down the drillstring with the opposite sign. When this wave reaches the bottom it is sometimes sufficient to cause the drill bit to reach zero velocity and be 'recaptured' by the frictional contact, as seen in case (b). The digital filter and finite element models show good agreement, with only small differences of detail: for case (a) this translates to a small difference in predicted sticking duration; and for case (b) this causes the stick-slip oscillation period to be slightly different, hence the apparently large peak errors for these figures is misleading. So it seems that the emergent features of the predictions are consistent and follow the same trends, and the differences give some indication of the reliability of the quantitative properties of the predictions.

Each feature of Figure 8 can be approximated analytically. The delay before initial slip $\tau_{1}$ is dominated by the time taken for the drillstring torque to reach its limiting value, and can be estimated using:

$$
\tau_{1}=\frac{T_{s}}{\Omega k_{\text {eff }}}
$$

where $T_{s}$ is the static torque limit, $\Omega$ is the steady surface angular velocity, $k_{\text {eff }}$ is the effective static torsional stiffness of the whole drillstring. It is assumed that the static torque limit is $20 \%$ larger than the dynamic slipping torque $T_{d}$. For this drillstring then case (a) gives $\tau_{1} \approx 3.5$ seconds, and case (b) gives $\tau_{1} \approx 14.2$ seconds. These estimates can be seen to be in good agreement with the numerical simulations.

For case (b) where the system begins stick-slip oscillation, the subsequent slip duration $\tau_{2}$ can be estimated as the time taken for a torsion wave to travel to surface and back. This is easily estimated from the torsional group velocity $v_{g}=3.2 \mathrm{kms}^{-1}$ and the total drillstring length $L_{\mathrm{t}}=1 \mathrm{~km}$, i.e. $\tau_{2}=L_{\mathrm{t}} / v_{g} \approx$ 0.6 seconds. Finally the sticking duration $\tau_{3}$ can be estimated as the time taken for the frictional torque to ramp up from the dynamic to the static frictional torque limits:

$$
\tau_{3}=\left[T_{s}-T_{d}\right] \Omega k_{\mathrm{eff}}
$$


For case $(\mathrm{b})$ this is estimated to be $\tau_{3} \approx 2.4$ seconds. This regime of frictioninduced vibration corresponds to a 'relaxation oscillation' in the friction literature (e.g. Putelat [25]).

Finally it is possible to estimate the stability boundary using a simple criterion for this kind of stick-slip oscillation. Stick-slip can only occur if the reverse torsional wave that is reflected from the surface is large enough to cause the drillbit to reach zero velocity. This can be estimated by determining whether or not the minimum value of the step response is greater than the surface RPM. In other words, stick-slip oscillation is expected to be able to be sustained if:

$$
\Omega<\left[T_{s}-T_{d}\right] \Delta
$$

where $\Delta$ is the largest undershoot of the unit step response at the drillbit:

$$
\Delta=-\left[\min _{t>0}\left(\int_{0}^{t} d_{N N}^{(t)} \mathrm{d} t\right)\right]
$$

A parametric study was carried out to identify the operating parameter space over which stick-slip can be sustained. Two operating parameters are varied: the drilling speed and the weight-on-bit (which maps to a dynamic torque limit $T_{d}$ according to Figure 4(a) and assuming drilling through sandstone). A summary of results is shown in Figure 9: the case studies above are shown as triangles; crosses indicate time-domain simulations that did not sustain stickslip; circles show cases that did stick-slip. Note that the parameter space is not very representative of industry practice, as the selected case study is for an academic geometry and results are intended to be illustrative of trends and to demonstrate the capability of the modelling approach. For each simulation both the digital filter and finite element methods predicted the same type of behaviour. The solid line shows the analytic boundary from Equation 74 and mapped to weight-on-bit using Figure 4(a) (sandstone), which is in excellent agreement with numerical simulations. It is clear that stick-slip oscillation can be sustained at slow drilling speeds and with large weight-on-bit. This is consistent with field observation and provides proof-of-concept for a tool to further explore parameter dependencies of the stick-slip boundary.

With this setup the filters took approximately 10 seconds to compute, and a 10-second time domain simulation coupled to a single-contact nonlinearity took approximately 120 seconds to compute. The finite element matrices took 5 seconds to compute and an equivalent simulation took 60 seconds. This demonstrates the efficiency of the approach and allows fast exploration of different situations. 


\subsubsection{Transient Lateral Excitation}

The next logical step is to simulate a lateral transient disturbance at the drillbit which causes side-contact with the borehole wall (in the absence of cutting). Two inputs are applied: a step change of 60 RPM in surface angular velocity at $t=0$ seconds, followed by a band-limited lateral impulse applied at $t=1$ second, with duration 0.02 seconds and amplitude $I=3 I_{0}$, where $I_{0}$ is the amplitude required for the drillstring to just make contact with the borehole wall. A Coulomb friction law is assumed at the interface between the drillstring and borehole wall, without distinguishing between static and sliding coefficients of friction. The coefficient of friction is taken to be $\mu_{0}=0.5$. Figure 10 shows the digital filter and finite element predictions for (a) the displacement from the centre of the borehole to the centre of the drillstring and (b) the trace of the centre of the drillstring. The dashed line represents the clearance radius in both (a) and (b). It can be seen that the impulse causes a sudden deviation from the centre of the borehole which initiates contact with the borehole wall. The rotating drillstring and the frictional contact then induce motion in the negative $y$ direction, but the conditions are not right for sustained backward whirl and the drillstring begins to return to the borehole centre after approximately 1-2 seconds of contact. It can be seen that the two methods give good agreement, where the main difference is again most likely due the difference in order of integration scheme between the digital filter and Finite Element models.

The simulation results shown in Figure 10 use a finite contact stiffness coefficient $k_{c}=1 \times 10^{9}$ : it was noted in Section 2.3.2 that a finite contact stiffness is not a numerical convenience and the case of infinite contact stiffness is readily computed. This is because the first coefficient of the discretised digital filters is non-zero which prevents a singularity in the contact force. To demonstrate, Figure 11 shows the infinite contact stiffness case equivalent to Figure 10. As expected, it can be seen that there is no longer any penetration into the borehole wall but that otherwise the behaviour is very similar: a period of high-frequency intermittent contact before borehole contact is lost. The remainder of simulations use a finite contact stiffness, but this choice is physics-based rather than for numerical convenience and for all cases tested the infinite contact stiffness simulations do not differ substantively.

Figure 12 shows a similar comparison using a higher coefficient of friction $\left(\mu_{0}=2\right)$ and keeping the impulse amplitude the same $\left(I=3 I_{0}\right)$. From (a) it can be seen that contact is maintained after the initial impact, and from (b) close inspection reveals the direction of travel of the centre of the drillstring is clockwise which is consistent with backward whirl as the drillstring itself is rotating anticlockwise. The details of the transients differ somewhat in each prediction but the overall features are similar. It can also be seen that the equilibrium backward whirl radius is slightly larger for the digital filter pre- 
dictions which is most likely to be due to the difference in integration schemes: the digital filter method uses a simple first-order scheme, while the finite element uses a second-order scheme. Despite differences in detail, the emergent behaviour is reassuringly similar for both simulation methods, and suggests that broad features of the predictions are robust.

Both models assume that nonlinearities are localised with potential contact locations that have been identified in advance. The finite element model readily allows the whole drillstring response to be reconstructed (it is also possible using the digital filters approach but more cumbersome). Figure 13 shows the peak lateral displacement along the length of the drillstring for the two example simulations shown in Figure 10 and 12. In both of these cases it can be seen that the maximum response does occur at the site of the nonlinearity and the local nonlinearity assumption is valid if the borehole clearance is assumed to be constant at $15 \mathrm{~mm}$. However, other scenarios could be envisaged where the borehole clearance is unexpectedly exceeded. An adaptive substructuring strategy could be developed for handling the class of problem where contact locations are unknown, but this is beyond the scope of the present study.

It is natural to use the model to explore the initiation conditions for backward whirl. A Monte Carlo test was carried out varying impulse amplitude and the coefficient of friction over the ranges $0.9 I_{0}<I<5 I_{0}$ and $0.5<\mu_{0}<3$. Figure 14 shows a summary of the results: ' $x$ ' backward whirl was not sustained in either model; ' $\square$ ' backward whirl was predicted by the finite element model but not the digital filter model; ' $O$ ' backward whirl was predicted by both models. The two triangles represent the examples shown above in Figures 10 and 12. It can be seen that agreement between the two methods is good where only a narrow band of cases gave different results (' $\square$ '). For this test system, a single lateral impulse only triggers backwards whirl if the coefficient of friction is large $\left(\mu_{0}>1\right)$. This is perhaps unsurprising as the impulse leads to an orthogonal impact with the borehole wall, so frictional forces would need to be very strong to push the system into a whirling state (tangential motion) before rebound occurs. Although there is a clear boundary it is not obvious how this might be predicted in advance: these simulations demonstrate a proof-of-concept efficient simulation approach that allows some progress to be made.

\subsubsection{Eccentricities}

The effect of drillpipe (or drillcollar) eccentricities can be accounted for in an ad-hoc fashion by assuming that the eccentricity is a lumped mass $m_{e}$ with offset $d$. It is further assumed that the dominant force acts in the radial direction with magnitude $m_{e}(d+r) \dot{\theta}^{2}$, where $r$ is the distance from the centre of the borehole to the centre of the drillstring. This force is correct for steady- 
state forward whirl and although eccentricity could be accounted for more carefully to include coriolis terms, it is hypothesised that its main effect is to cause forward whirl. Therefore these simple assumptions are expected to capture the main effects, even if the transient details are not fully captured.

By way of example, it is assumed that there is an eccentricity of $m_{e} d=$ $100 \mathrm{kgm}$ at the end of the drillpipe, and the input surface angular velocity is taken to be a step of 60 RPM as before. Figure 15(a) shows the drillstring displacement from centre, and (b) shows the $x-y$ trace. It can be seen that the eccentricity causes contact to be made with the borehole wall, then frictional forces cause an initial transient which is in the opposite direction to the drillstring rotation but that the eccentric force dominates leading to forward whirl. There is broad agreement between the two predictions, with small differences visible in the details of the transients and equilibrium radius.

\subsection{Multi-Contact Behaviour}

In all of the above examples it has been assumed that nonlinearity is confined to a single location: a multi-contact scenario is now considered. It is assumed that contact can occur at three downhole sites: $100 \mathrm{~m}, 50 \mathrm{~m}$ and $0 \mathrm{~m}$ from the drillbit corresponding to Sites (1), (2) and (3) respectively. It is assumed that these sites represent stabiliser locations where the clearance is at a minimum. The surface angular velocity profile is a step of $60 \mathrm{RPM}$, and at $t=1$ second a lateral impulse is applied at Site (2). The side-wall coefficient of friction is taken to be $\mu_{0}=0.5$. This all represents an artificial test case: the purpose is simply to provide proof of concept for the applicability of the model to handle multiple nonlinearities.

Figure 16 shows the displacement amplitude for two example simulations. The left-side plots (a,c,e) show the predictions for Sites (1), (2), and (3) respectively with $\mu_{0}=0.5$. The right-side plots $(\mathrm{b}, \mathrm{d}, \mathrm{f})$ show the equivalent predictions with $\mu_{0}=2$. It can be seen in $(\mathrm{a}, \mathrm{c}, \mathrm{e})$ with $\mu_{0}=0.5$ that side-wall contact is not sustained while in $(b, d, f)$ backward whirl is initiated. The behaviour is similar to the single contact case shown in Figure 12, but in this multi-contact scenario the transient behaviour is more complicated: the disturbance impulse causes impact events at all three sites and the resulting whirling behaviour is not steady, particularly in (f) it can be seen that the drillstring intermittently loses contact with the borehole wall.

Figure 17 shows a parametric study investigating the initiation conditions of backward whirl, equivalent to the single-contact study shown in Figure 14. The results are similar to the previous single-contact case: high amplitude disturbances together with a high coefficient of friction consistently lead to 
sustained backward whirl. Squares indicate cases for which the finite element model predicted backward whirl, but the digital filter method did not. In most cases the discrepancies fall on an obvious boundary as before. On closer inspection it can be seen that the boundary in this case is slightly lower than for the single contact case: this is perhaps due to the additional contacts resulting in a higher effective frictional force which tend to push the system towards backward whirl. It would be interesting to explore how this boundary moves as the number of allowed contact points increases and in the limit as distributed contact is considered: however, this falls beyond the scope of the present study.

\subsection{Computational Costs}

Both the digital filter and the Finite Element models are based on a discretisation of the drillstring and a reduction to 'nonlinear' degrees of freedom. The digital filter approach is based on discretising in time the impulse response functions corresponding to the nonlinear degrees of freedom. Finding the impulse response functions represents a fixed overhead computational cost: the filters for the multi-contact test case above took approximately 10 seconds on a laptop (late-2011 macbook pro, quad core $2.5 \mathrm{GHz}$ Intel Core i7). This calculation could be carried out once for a given set of parameters characterising the linear dynamics of the drillstring. On average, a 10 second simulation with $f_{s} \approx 640 \mathrm{~Hz}$ and with three sites of nonlinearity took approximately $250 \mathrm{sec}-$ onds to compute. The memory required for the multi-contact test case above was very small at around $20 \mathrm{MB}$, including all variables used for a 10 second simulation and storage of results.

The Finite Element model is based on discretising the drillstring in space to generate mass, damping and stiffness matrices. Calculation of these matrices represents a fixed overhead cost: the matrices for the multi-contact test case above took approximately 6 seconds. On average, a 10 second simulation with three sites of nonlinearity took approximately 150 seconds to compute. The memory required was small at around $120 \mathrm{MB}$, including all variables and storage of results.

The differences in computation time and required memory are not significant, as they are most likely due to particular choices of implementation in the Matlab code and also choice of discretisation resolution for the two methods. Fundamentally the two methods have rather similar requirements, which is most obvious from the similarity of the time-stepping equations for each method: Equations 61 and 68. 


\section{Concluding Remarks}

Two efficient models for predicting the nonlinear dynamics of the full length of a drillstring have been presented: (1) using a digital filter approach; and (2) using the finite element method. In both methods efficiency is achieved by assuming that the dominant sources of nonlinearity are spatially localised, allowing reduction of the system to the nonlinear degrees of freedom.

The method was applied to a $1 \mathrm{~km}$ drillstring and was shown to be able to efficiently demonstrate a range of phenomena observed in the field: stick-slip oscillation; forward whirl and backward whirl. Parametric studies confirmed field observations that stick-slip oscillation is sustained at low drilling speeds and high weight-on-bit, and a simple analytic criterion for the stability boundary was derived and found to be in good agreement with numerical simulations. The initial conditions of backward whirl were explored and appeared to reveal a bound for the coefficient of friction, below which backward whirl could not be initiated. As the coefficient of friction was increased, a smaller amplitude disturbance was able to initiate backward whirl.

A multi-contact scenario was presented which demonstrated similar behaviour for disturbance-initiated backward whirl. A slightly lower coefficient of friction and disturbance amplitude were found to initiate backward whirl, but the effect was relatively small. The initial parameter studies carried out motivate further research into the operating conditions that lead to high amplitude vibration regimes: this is the subject of ongoing research.

All simulations were carried out using both the digital filter and finite element methods: good agreement was observed in all cases, particularly in terms of the emergent qualitative behaviour of the system. Differences in the details of the predictions were observed, giving some indication as to the precision of predictions.

Both methods were found to be extremely efficient and could still predict key phenomena commonly observed in drillstring dynamics, suggesting that the local nonlinearity approximation may be appropriate for further parametric investigation of this class of problem. The key advantages of the digital filter approach are that the impulse response characterisation provides more physical intuition of the system behaviour, and the relevant impulse responses could potentially be identified using a variety of methods (numerical or perhaps experimental). The main advantage of the Finite Element formulation is that it is a more commonly used framework which makes it more straightforward to apply to existing models. 


\begin{tabular}{|c|c|}
\hline Symbol & Definition \\
\hline BHA & Bottom Hole Assembly (end section of drillstring) \\
\hline $\mathrm{DOC}$ & Depth of cut per revolution (m) \\
\hline ROP & Rate of penetration $\left(\mathrm{ms}^{-1}\right)$ \\
\hline $\mathrm{RPM}$ & Revolutions per minute \\
\hline WOB & Weight on bit $(\mathrm{N})$ \\
\hline hilb & Discrete Hilbert Transform \\
\hline IDFT & Inverse Discrete Fourier Transform \\
\hline$\bullet$ & Complex conjugate \\
\hline$\bullet^{T}$ & Transpose \\
\hline$\bullet$ & Frequency domain variable \\
\hline$\dot{\bullet}$ & Differentiation with respect to time \\
\hline$\bullet^{\prime}$ & Differentiation with respect to distance along drillstring \\
\hline$\bullet_{h}$ & 'History' term: convolution involving only past states \\
\hline$*$ & Convolution operation \\
\hline A & Overall impulse response matrix \\
\hline a & Contributions of eigenvectors to solution \\
\hline$A$ & Drillstring cross-sectional area $\left(\mathrm{m}^{2}\right)$ \\
\hline$C$ & Drillstring pre-compression $(\mathrm{N})$ \\
\hline $\mathbf{C}^{(b)}$ & Finite Element damping matrix (bending) \\
\hline$c_{b}$ & Damping coefficient for bending $\left(\mathrm{Nsm}^{-2}\right)$ \\
\hline$c_{t}$ & Damping coefficient for torsion $\left(\mathrm{Nmsrad}^{-1}\right)$ \\
\hline $\mathbf{C}^{(t)}$ & Finite Element damping matrix (torsion) \\
\hline $\mathbf{D}^{(b)}$ & Input-output frequency domain matrix (bending) \\
\hline $\mathbf{D}^{(t)}$ & Input-output frequency domain matrix (torsion) \\
\hline $\mathrm{E}$ & Overall vector of inputs \\
\hline$E$ & Young's modulus (GPa) \\
\hline$f_{s}$ & Sampling frequency $(\mathrm{Hz})$ \\
\hline
\end{tabular}




\begin{tabular}{|c|c|}
\hline $\mathbf{F}$ & Combined vector of lateral forces $(\mathrm{N})$ \\
\hline$F_{F}$ & $\begin{array}{l}\text { Total external force in frictional (tangential) direction } \\
(\mathrm{N})\end{array}$ \\
\hline $\mathbf{F}_{\mathbf{I}}$ & External force disturbance vector $(\mathrm{N})$ \\
\hline$F_{N}$ & $\begin{array}{l}\text { External force contribution from contact in normal direc- } \\
\text { tion }(\mathrm{N})\end{array}$ \\
\hline$F_{R}$ & Total external force in radial (normal) direction $(\mathrm{N})$ \\
\hline$F_{T}$ & $\begin{array}{l}\text { External force contribution from contact in tangential } \\
\text { direction }(\mathrm{N})\end{array}$ \\
\hline$F_{x}$ & Total external force in $x$-direction $(\mathrm{N})$ \\
\hline $\mathbf{F}_{x}$ & Vector of external forces in $x$-direction (N) \\
\hline$F_{y}$ & Total external force in $y$-direction $(\mathrm{N})$ \\
\hline $\mathbf{F}_{y}$ & Vector of external forces in $y$-direction $(\mathrm{m})$ \\
\hline$G$ & Shear modulus (GPa) \\
\hline $\mathbf{G}(t)$ & Matrix of impulse response functions (torsion) \\
\hline $\mathbf{H}(t)$ & Matrix of impulse response functions (bending) \\
\hline$\overline{\mathbf{G}}(j \omega)$ & Matrix of transfer functions (torsion) \\
\hline$\overline{\mathbf{H}}(j \omega)$ & Matrix of transfer functions (bending) \\
\hline$I$ & Second moment of area $\left(\mathrm{m}^{4}\right)$ \\
\hline$I_{0}$ & $\begin{array}{l}\text { Impulse amplitude needed to just initiate borehole wall } \\
\text { contact }(\mathrm{N})\end{array}$ \\
\hline$I$ & Impulse amplitude normalised to $I_{0}$ \\
\hline$J$ & Polar moment of inertia $\left(\mathrm{kgm}^{2}\right)$ \\
\hline$k$ & Time index \\
\hline$K$ & $\begin{array}{l}\text { Effective linearised feedback gain for digital filter stabil- } \\
\text { ity analysis }\end{array}$ \\
\hline $\mathbf{K}^{(b)}$ & Dynamic stiffness matrix (bending) \\
\hline $\mathbf{K}^{(b)}$ & Finite Element stiffness matrix (bending) \\
\hline $\mathbf{K}_{\text {eff }}^{(b)}$ & $\begin{array}{l}\text { Effective stiffness from Newmark-Beta integration (bend- } \\
\text { ing) }\end{array}$ \\
\hline$k_{c}$ & Contact stiffness coefficient $\left(\mathrm{Nm}^{-1}\right)$ \\
\hline
\end{tabular}



$\mathbf{K}^{(t)} \quad$ Dynamic stiffness matrix (torsion)
$\mathbf{K}^{(t)} \quad$ Finite Element stiffness matrix (torsion)
$\mathbf{K}_{\text {eff }}^{(t)} \quad$ Effective stiffness from Newmark-Beta integration (tor- sion)
$L_{1,2} \quad$ Length of (1) pipe and (2) joint section of drillstring (m)
$\mathbf{M}_{n}^{(t)} \quad$ Transfer matrix for the $n$th pipe and joint section (tor- sion)
$\mathbf{M}_{n}^{(b)} \quad$ Transfer matrix for the $n$th pipe and joint section (bend- ing)
$M_{m n} \quad$ Internal bending moment at interface between two sec- tions of drillstring $(\mathrm{Nm})$
$\mathbf{M}^{(b)} \quad$ Finite Element mass matrix (bending)
$m_{e} \quad$ Eccentric mass $(\mathrm{kg})$
$M_{n}^{(e x t)} \quad$ External bending moment at end of $n$ section of drill- string $(\mathrm{Nm})$
$\mathbf{M}^{(t)} \quad$ Finite Element mass matrix (torsion)
$P \quad$ Top partition of $\boldsymbol{\Phi}$
$Q \quad$ Bottom partition of $\boldsymbol{\Phi}$
$R_{i} \quad$ Magnitude of $i$ th eigenvalue of $\mathbf{M}_{n}^{(t, b)}$
$R_{n} \quad$ Radial displacement of $n$ section of drillstring
$r_{1,2} \quad$ Internal radius of (1) pipe and (2) joint section of drill- string $(\mathrm{m})$
$R_{1,2} \quad$ External radius of (1) pipe and (2) joint section of drill- string $(\mathrm{m})$
S Scaling matrix to prevent ill-conditioning
$S_{m n} \quad$ Internal shear at interface between two sections of drill- string $(\mathrm{N})$
$S_{n}^{(e x t)} \quad$ External shear force at end of $n$ section of drillstring (N)
$T_{i} \quad$ Torque at end of $i$ th pipe and joint section (Nm)
$t \quad$ Time $(\mathrm{s})$
$\mathbf{T}^{(e x t)} \quad$ External torque vector $(\mathrm{Nm})$
$T_{s}, T_{d} \quad$ Static and dynamic friction torque limits $(\mathrm{Nm})$ 


\section{$\mathrm{U}$}

$v_{g}$

$\mathbf{v}$

$V_{s}$

W

$x$

$\mathrm{x}$

y

$\mathbf{y}$

$\mathbf{z}$

$\alpha, \beta$

$\delta$

$\Delta$

$\Gamma^{(b)}$

$\Gamma^{(t)}$

$\Lambda$

$\lambda$

$\mu_{0}$

$\omega$

$\Phi$

$\Psi$

$\rho$

$\tau_{i}$

$\theta, \dot{\theta}, \ddot{\theta}$

$\xi$
Overall vector of outputs

Group velocity $\left(\mathrm{ms}^{-1}\right)$

Augmentation vector

Side-wall sliding velocity $\left(\mathrm{ms}^{-1}\right)$

Localised self-weight $(\mathrm{N})$

Displacement (m)

Vector of $x$-displacements (m)

Displacement $(\mathrm{m})$

Vector of $y$-displacements (m)

Combined vector of lateral displacements (m)

Newmark-Beta integration parameters

Contact penetration (m)

Largest undershoot of unit driving point step response at drillbit $(\mathrm{m})$

First order matrix for bending

First order matrix for torsion

Eigenvalues of $\mathbf{M}_{n}^{(t)}$

Lagrange multiplier

Side-wall coefficient of friction

Frequency $\left(\operatorname{rads}^{-1}\right)$

Right eigenvectors of $\mathbf{M}_{n}^{(t)}$

Left eigenvectors of $\mathbf{M}_{n}^{(t)}$

Density $\left(\mathrm{kgm}^{-3}\right)$

Stick-slip characteristic times (s)

Angular position, velocity and acceleration $\left(\mathrm{rad}, \mathrm{rads}^{-1}\right.$, $\operatorname{rads}^{-2}$ )

Distance along drillstring from surface $(\mathrm{m})$ 


\section{Acknowledgements}

The authors are grateful to industrial support and permission to publish this work, and would like to thank Prof Jim Woodhouse for technical contributions, and Dr Louis Kovalevski for assistance with the Finite Element model.

\section{References}

[1] S. Jardine, D. Malone, and M. Sheppard. Putting a damper on drilling's bad vibrations. Oilfield Review, 1994.

[2] T. Richard, C. Germay, and E. Detournay. Self-excited stick-slip oscillations of drill bits. Comptes Rendus Mécanique, 332(8):619-626, August 2004.

[3] T. Richard, C. Germay, and E. Detournay. A simplified model to explore the root cause of stickslip vibrations in drilling systems with drag bits. Journal of Sound and Vibration, 305(3):432-456, August 2007.

[4] Y. Kovalyshen. A simple model of bit whirl for deep drilling applications. Journal of Sound and Vibration, 332(24):6321-6334, November 2013.

[5] A. Depouhon and E. Detournay. Instability regimes and self-excited vibrations in deep drilling systems. Journal of Sound and Vibration, 333(7):2019-2039, March 2014.

[6] Y.A. Khulief and H. Al-Naser. Finite element dynamic analysis of drillstrings. Finite Elements in Analysis and Design, 41(13):1270-1288, July 2005.

[7] A.P. Christoforou and A.S. Yigit. Fully coupled vibrations of actively controlled drillstrings. Journal of Sound and Vibration, 267(5):1029-1045, November 2003.

[8] C. Germay, V. Denoël, and E. Detournay. Multiple mode analysis of the selfexcited vibrations of rotary drilling systems. Journal of Sound and Vibration, 325:362-381, 2009.

[9] X. Lui, N. Vlajic, X. Long, G. Meng, and B. Balachandran. State-dependent delay influenced drill-string oscillations and stability analysis. ASME Journal of Vibration and Acoustics, 136(5), 2013.

[10] R. I. Leine and D. H. Van Campen. Stick-slip whirl interaction in drillstring dynamics. In Solid Mechanics and its Applications, volume 122, pages 287-296, 2005 .

[11] A. S. Yigit and A. P. Christoforou. Stick-Slip and Bit-Bounce Interaction in Oil-Well Drillstrings. Journal of Energy Resources Technology, 128(4):268, 2006.

[12] Y. Kovalyshen. Understanding root cause of stickslip vibrations in deep drilling with drag bits. International Journal of Non-Linear Mechanics, 67:331-341, December 2014. 
[13] S. A. Tobias. Machine-Tool Vibration. Blackie \& Son Limited, 1965.

[14] H. E. Merritt. Theory of Self-Excited Machine-Tool Chatter. Journal of Engineering for Industry, 87(4):447, 1965.

[15] M.E. McIntyre and J. Woodhouse. On the Fundamentals of Bowed-String Dynamics. Acustica, 43:93-108, 1979.

[16] R. W. Clough and E. L. Wilson. Dynamic analysis of large structural systems with local nonlinearities. Computer Methods in Applied Mechanics and Engineering, 17-18:107-129, January 1979.

[17] R. S. Langley. A Transfer Matrix Analysis of the Energetics of Structural Wave Motion and Harmonic Vibration. In Proceedings of the Royal Society A: Mathematical, Physical and Engineering Sciences, volume 452, pages 16311648, 1996.

[18] R. Szalai. Impact mechanics of elastic bodies with point contact. arXiv:1306.2224v2 [math.DS], June 2013.

[19] S S. Rao. The finite element method in engineering. Elsevier Science \& Technology Books, 2005.

[20] R. V. Dukkipati, M. A. Rao, and R. B. Bhat. Computer aided analysis and design of machine elements. New Age International (P) Limited, Publishers, 2000 .

[21] V. A. Dunayevsky, F. Abbassian, and A. Judzis. Dynamic Stability of Drillstrings Under Fluctuating Weight on Bit. SPE Drilling 83 Completion, 8(2), 1993.

[22] Y.A. Khulief, F.A. Al-Sulaiman, and S. Bashmal. Vibration analysis of drillstrings with self-excited stick-slip oscillations. Journal of Sound and Vibration, 299(3):540-558, January 2007.

[23] E. Detournay, T. Richard, and M. Shepherd. Drilling response of drag bits: theory and experiment. International Journal of Rock Mechanics and Mining Sciences, 45:1347-1360, 2008.

[24] T. Butlin and R. S. Langley. An efficient model of drillstring dynamics with localised nonlinearities. In Proceedings of the IUTAM Symposium on the Vibration Analysis of Structures with Uncertainties, pages 389-401, St. Petersburg, 2009.

[25] T. Putelat, J. H. P. Dawes, and J. R. Willis. Regimes of frictional sliding of a spring-block system. Journal of the Mechanics and Physics of Solids, 58(1):2753, January 2010. 


\section{List of Figures}

1 Definition of variables for a pipe and joint section.

2 Cross-section of geometry and definition of variables during contact between the drillstring and borehole wall and definition of variables.

3 Block diagram for torsion with frictional feedback.

4 Relationship between weight-on-bit, torque and depth-of-cut for a 12 inch drillbit for sandstone (red dashed line) and shale (blue solid line): (a) torque; and (b) depth of cut per revolution.

5 Two test scenarios: (A) with a single nonlinearity at the drillbit (either a nonlinear cutting law or borehole wall contact); and (B) with three nonlinearities distributed along the BHA (including both cutting and side-wall contact nonlinearities).

6 Example transfer functions to illustrate the linear dynamics of the example test system: (a) surface to downhole angular velocity $\bar{G}_{N 0}(i \omega)$; and (b) downhole lateral force to displacement $\bar{H}_{N N}(i \omega)$.

7 Comparison of digital filter predictions with finite element predictions for the transient response of a linear drillstring: (a) downhole angular velocity response to $60 \mathrm{RPM}$ step change in surface velocity; and (b) downhole lateral response to unit impulse.

8 Example downhole transient response for a surface step input:

(a) $\Omega=0.2 \mathrm{RPM}, T_{\mathrm{d}}=2 \times 10^{3} \mathrm{Nm}$ (leading to smooth rotation) and (b) $\Omega=0.1 \mathrm{RPM}, T_{\mathrm{d}}=4 \times 10^{3} \mathrm{Nm}$ (leading to stick-slip oscillation).

$9 \quad$ Operating conditions leading to stick-slip oscillation (o) or smooth drilling $(x)$. Previous examples from Figure 8 from shown as triangles $(\boldsymbol{\Lambda})$. The solid line shows the predicted boundary from Equation 74 with torque mapped to weight-on-bit using Figure 4(a). 
10 Comparison of digital filter predictions with finite element predictions for the nonlinear transient response of the drillbit to an impulse of amplitude $I=3 I_{0}$ with side-wall coefficient of friction $\mu_{0}=0.5$ : (a) radial displacement as a function of time; and (b) $x-y$ trace of the displacement. The dashed line represents the borehole clearance radius. The drillstring makes temporary contact with the borehole wall.

11 Comparison of digital filter predictions with finite element predictions for the nonlinear transient response of the drillbit to an impulse of amplitude $I=3 I_{0}$ with side-wall coefficient of friction $\mu_{0}=0.5$ using an infinite contact stiffness: (a) radial displacement as a function of time; and (b) $x-y$ trace of the displacement. The dashed line represents the borehole clearance radius. The drillstring makes temporary contact with the borehole wall.

12 Comparison of digital filter predictions with finite element predictions for the nonlinear transient response of the drillstring to an impulse of amplitude $I=3 I_{0}$ with coefficient of friction $\left(\mu_{0}=2\right)$ : (a) radial displacement as a function of time; and (b) $x-y$ trace of the displacement. The dashed line represents the borehole clearance radius. The drillstring makes contact with the borehole wall which then leads to sustained backward whirl.

13 Reconstruction of peak lateral amplitude of whole drillstring response. The dashed line shows the transient contact simulation with coefficient of friction $\mu_{0}=0.5$ and the solid line shows the example backward whirl simulation with coefficient of friction $\mu_{0}=2$.

14 Initiation conditions for backwards whirl, varying impulse amplitude $I / I_{0}$ and coefficient of friction $\mu_{0}$ : sustained backwards whirl (circles); transient response (crosses); and discrepancies between finite element and digital filter predictions (squares).

15 Comparison of digital filter predictions with finite element predictions for the nonlinear transient response of the drillstring with an eccentricity of $m_{e} d=100 \mathrm{kgm}$ : (a) radial displacement as a function of time; and (b) $x-y$ trace of the displacement. The dashed line represents the borehole clearance radius. The drillstring makes contact with the borehole wall which then leads to sustained forwards whirl. 
16 Comparison of digital filter predictions with finite element predictions for the nonlinear transient response of the drillstring to an impulse of amplitude $I=3 I_{0}$. The left-side plots (a,c,e) are for a coefficient of friction $\mu_{0}=0.5$; while the right-side plots $(\mathrm{b}, \mathrm{d}, \mathrm{f})$ are for $\mu_{0}=2$. The pair $(\mathrm{a}, \mathrm{b})$ show the predicted response for Site (1); (c,d) show Site (2); and (e,f) show Site (3). The horizontal dashed line shows the amplitude

17 Initiation conditions for backwards whirl for a drillstring with three points of contact, varying impulse amplitude $I / I_{0}$ and coefficient of friction $\mu_{0}$ : sustained backwards whirl (circles); transient response (crosses); and discrepancies between finite element and digital filter predictions (squares). 


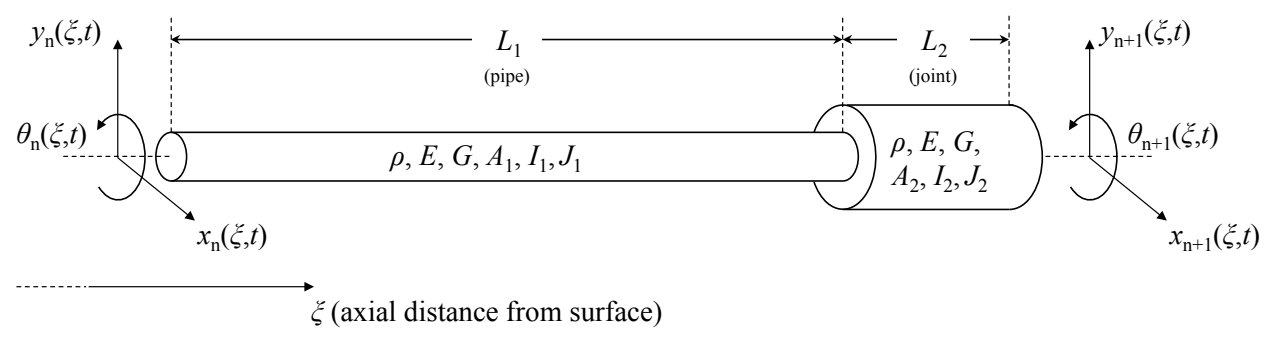

Fig. 1. Definition of variables for a pipe and joint section.

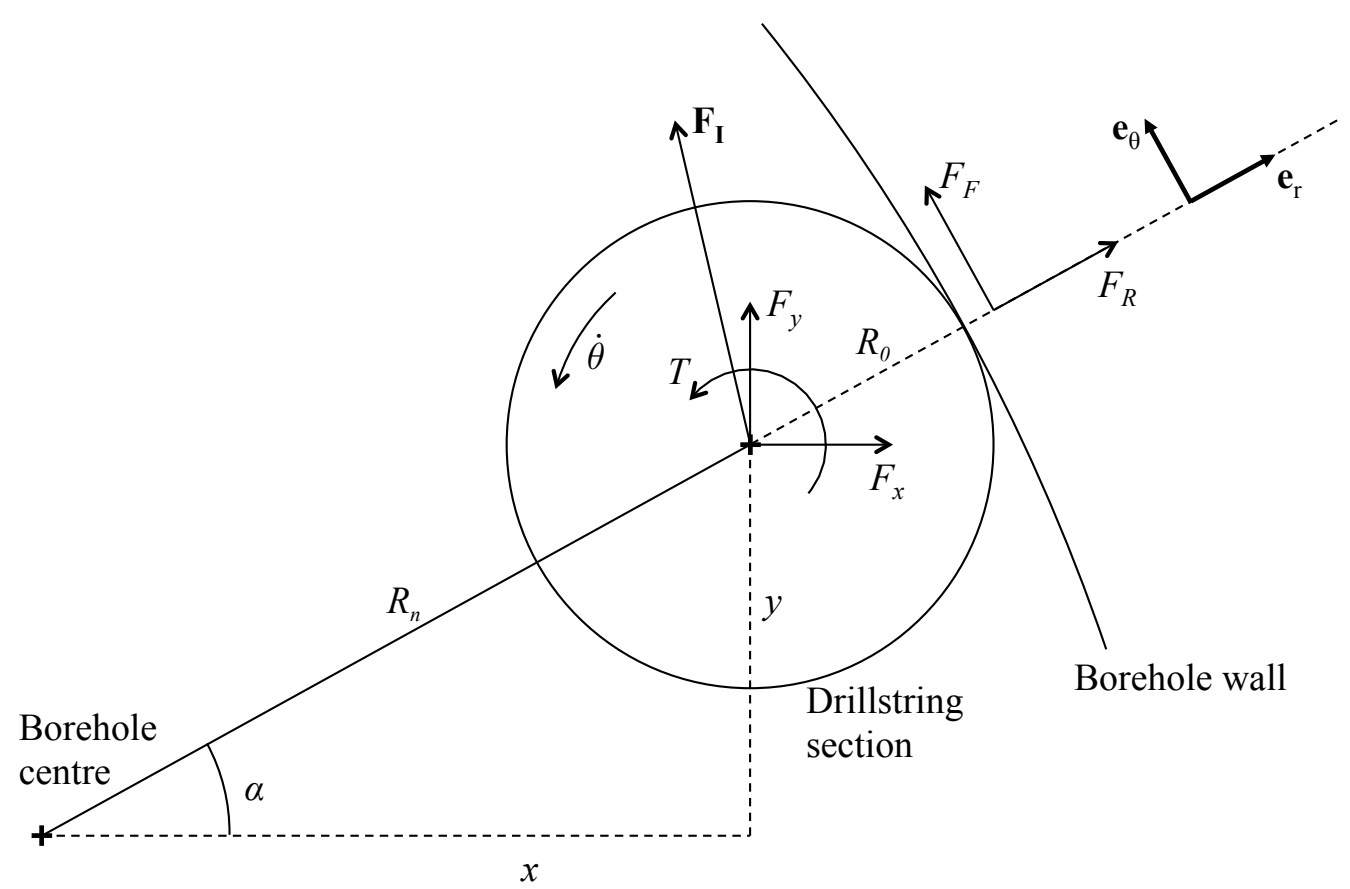

Fig. 2. Cross-section of geometry and definition of variables during contact between the drillstring and borehole wall and definition of variables. 


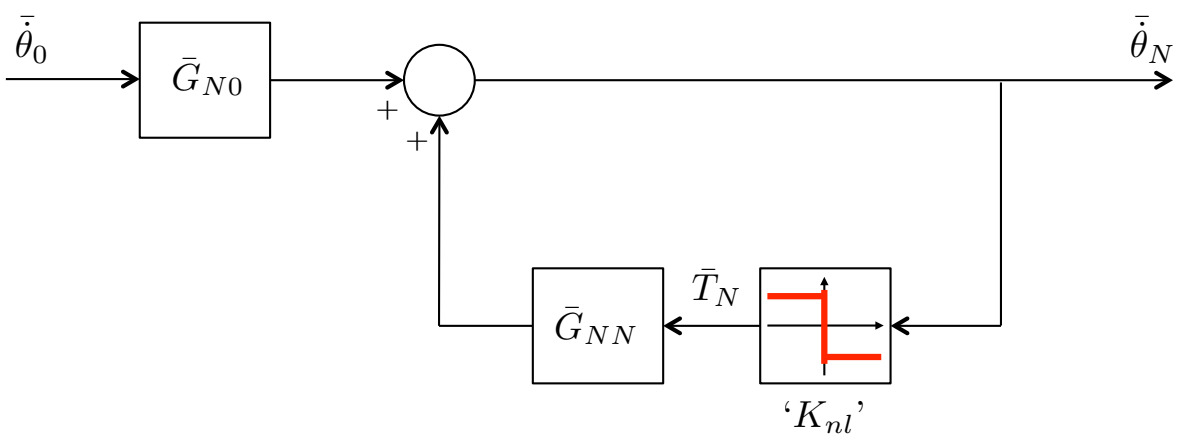

Fig. 3. Block diagram for torsion with frictional feedback.

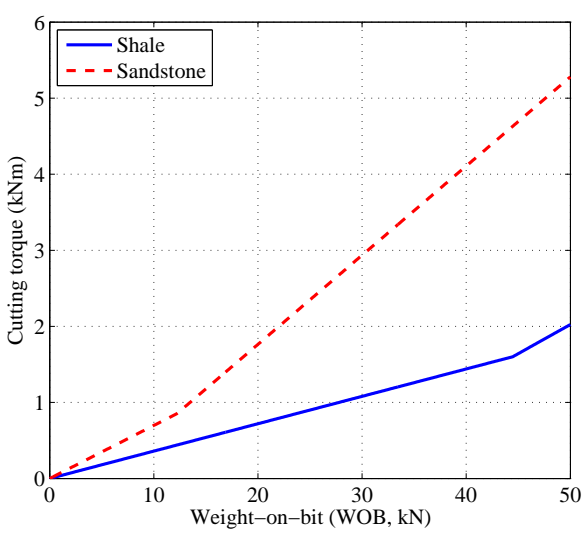

(a)

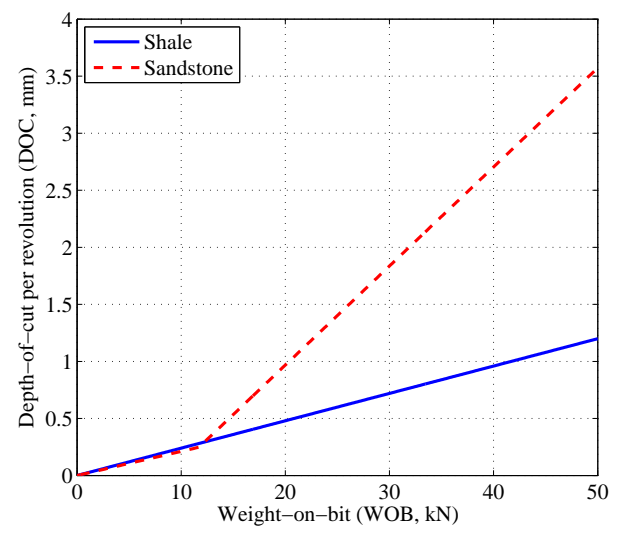

(b)

Fig. 4. Relationship between weight-on-bit, torque and depth-of-cut for a 12 inch drillbit for sandstone (red dashed line) and shale (blue solid line): (a) torque; and (b) depth of cut per revolution. 


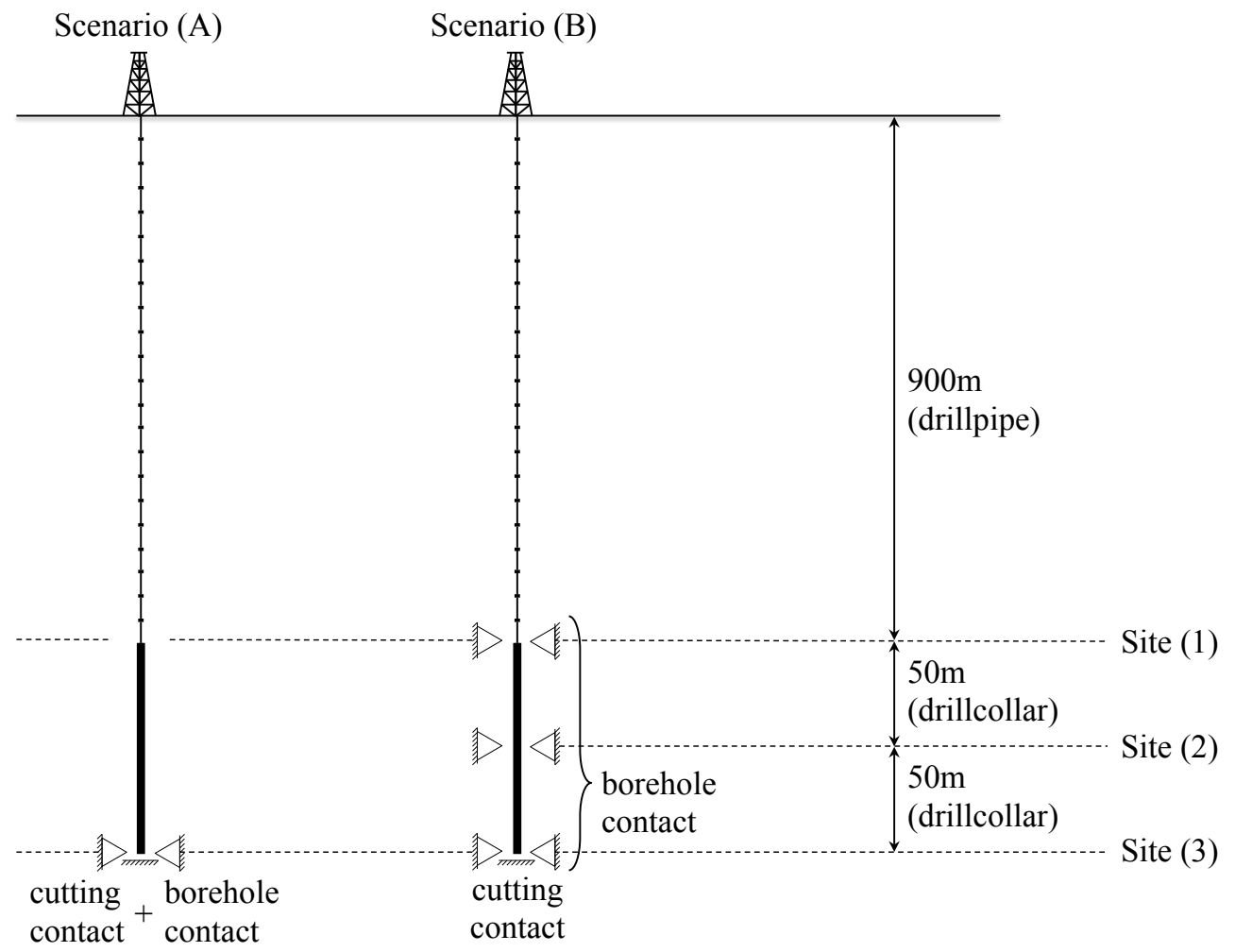

Fig. 5. Two test scenarios: (A) with a single nonlinearity at the drillbit (either a nonlinear cutting law or borehole wall contact); and (B) with three nonlinearities distributed along the BHA (including both cutting and side-wall contact nonlinearities).

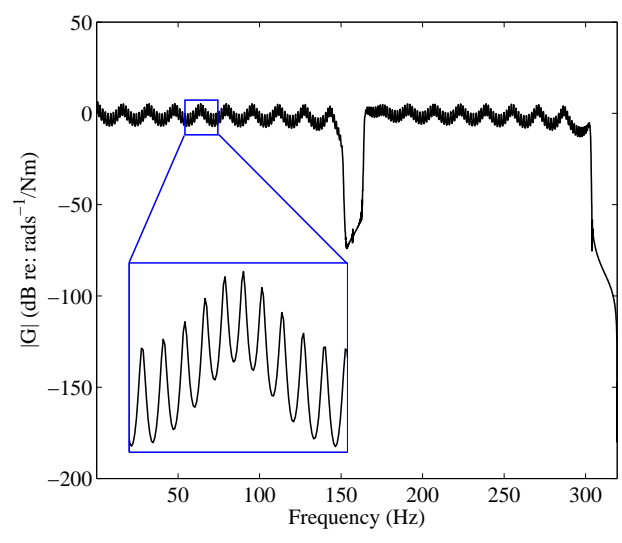

(a)

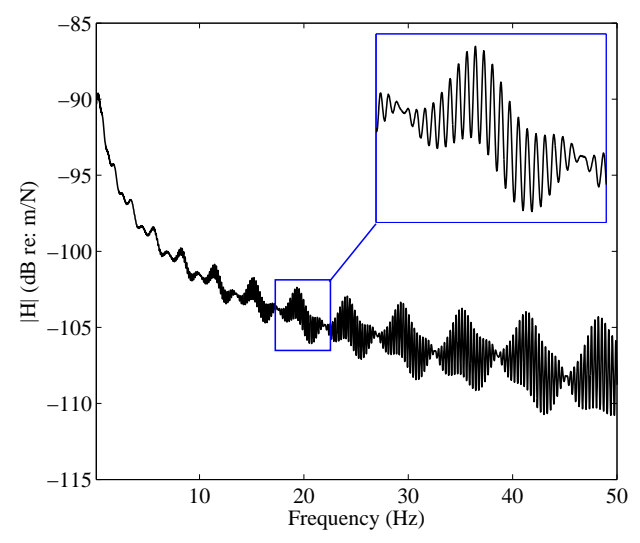

(b)

Fig. 6. Example transfer functions to illustrate the linear dynamics of the example test system: (a) surface to downhole angular velocity $\bar{G}_{N 0}(i \omega)$; and (b) downhole lateral force to displacement $\bar{H}_{N N}(i \omega)$. 


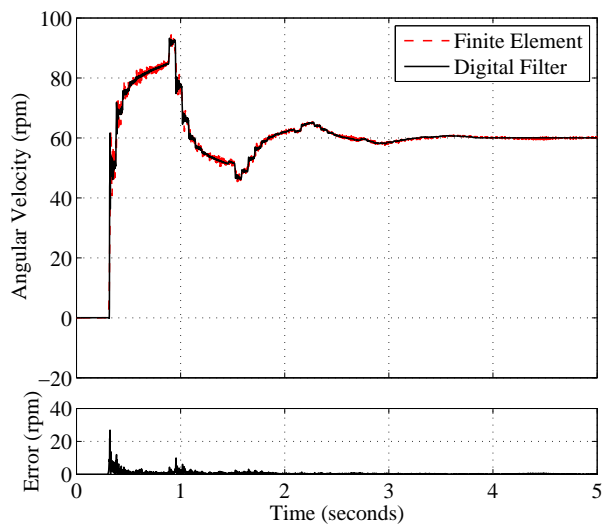

(a)

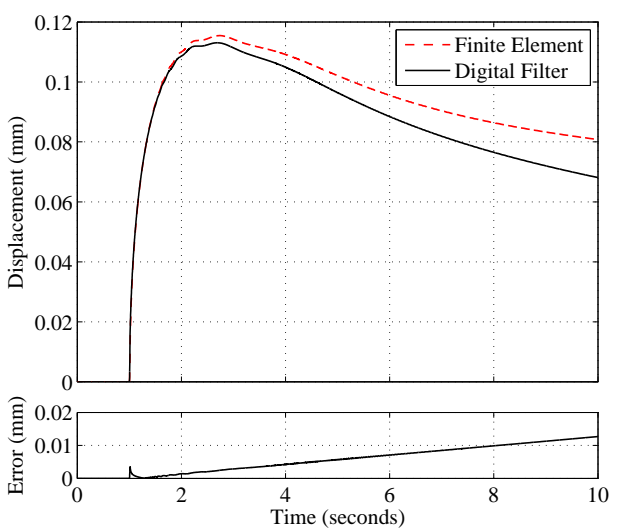

(b)

Fig. 7. Comparison of digital filter predictions with finite element predictions for the transient response of a linear drillstring: (a) downhole angular velocity response to 60 RPM step change in surface velocity; and (b) downhole lateral response to unit impulse.

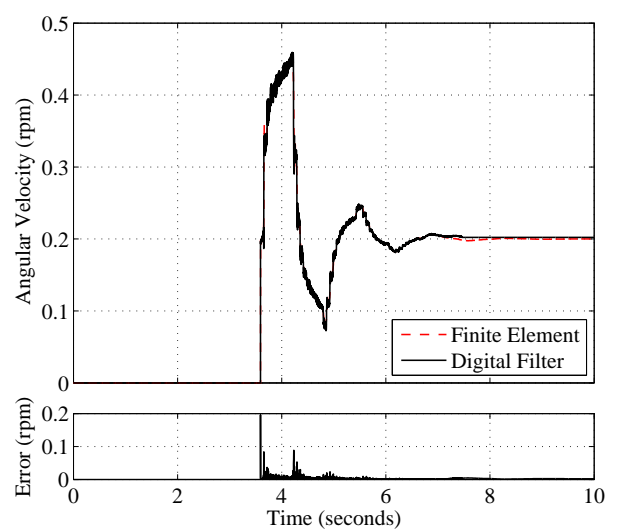

(a)

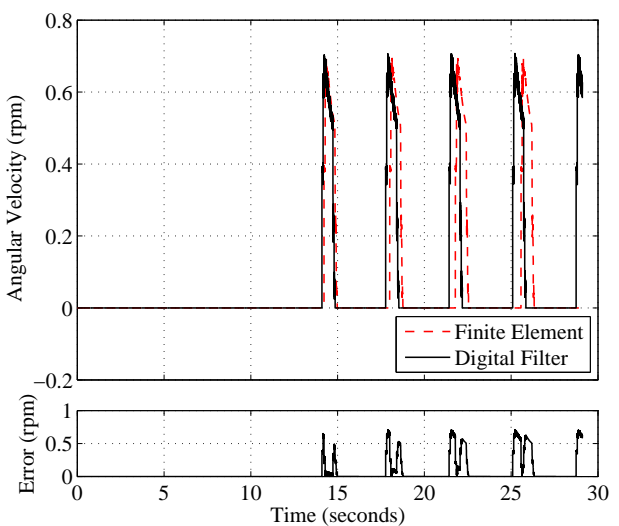

(b)

Fig. 8. Example downhole transient response for a surface step input: (a) $\Omega=0.2 \mathrm{RPM}, T_{\mathrm{d}}=2 \times 10^{3} \mathrm{Nm}$ (leading to smooth rotation) and (b) $\Omega=0.1 \mathrm{RPM}, T_{\mathrm{d}}=4 \times 10^{3} \mathrm{Nm}$ (leading to stick-slip oscillation). 


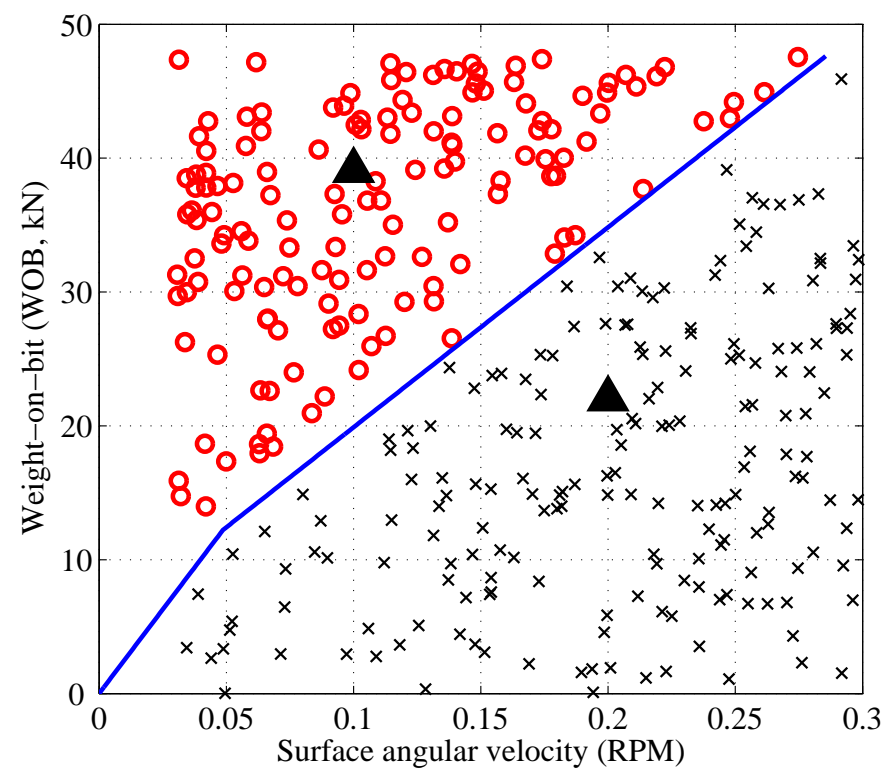

Fig. 9. Operating conditions leading to stick-slip oscillation (०) or smooth drilling $(\times)$. Previous examples from Figure 8 from shown as triangles $(\mathbf{\Lambda})$. The solid line shows the predicted boundary from Equation 74 with torque mapped to weight-on-bit using Figure 4(a).

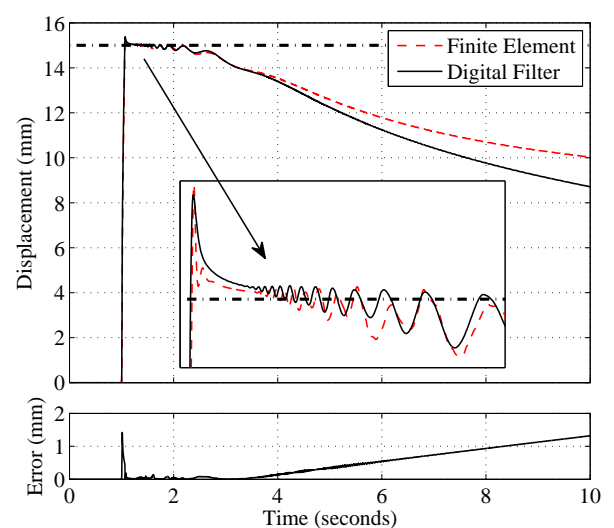

(a)

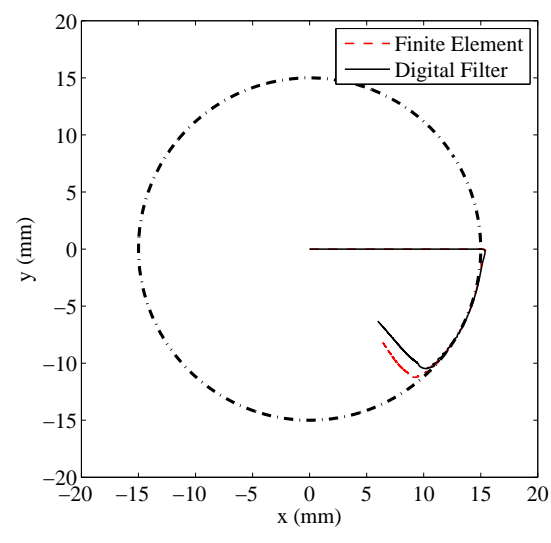

(b)

Fig. 10. Comparison of digital filter predictions with finite element predictions for the nonlinear transient response of the drillbit to an impulse of amplitude $I=3 I_{0}$ with side-wall coefficient of friction $\mu_{0}=0.5$ : (a) radial displacement as a function of time; and (b) $x-y$ trace of the displacement. The dashed line represents the borehole clearance radius. The drillstring makes temporary contact with the borehole wall. 


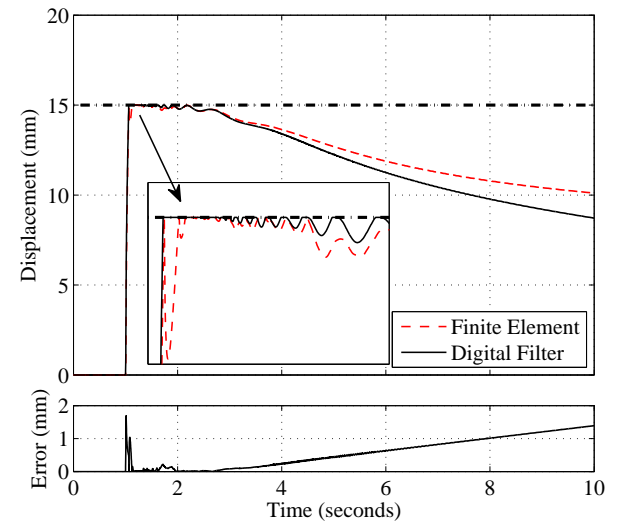

(a)

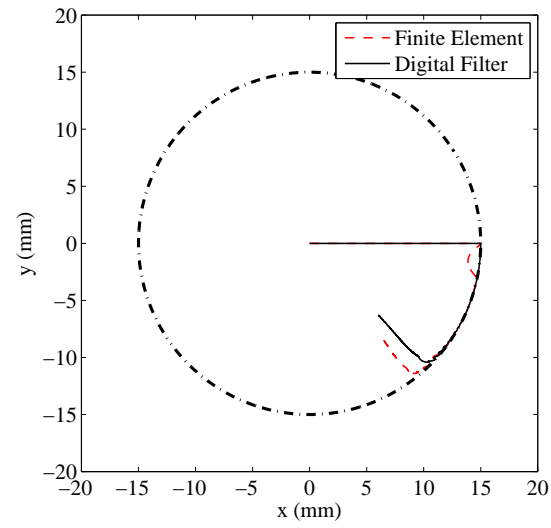

(b)

Fig. 11. Comparison of digital filter predictions with finite element predictions for the nonlinear transient response of the drillbit to an impulse of amplitude $I=3 I_{0}$ with side-wall coefficient of friction $\mu_{0}=0.5$ using an infinite contact stiffness: (a) radial displacement as a function of time; and (b) $x-y$ trace of the displacement. The dashed line represents the borehole clearance radius. The drillstring makes temporary contact with the borehole wall.

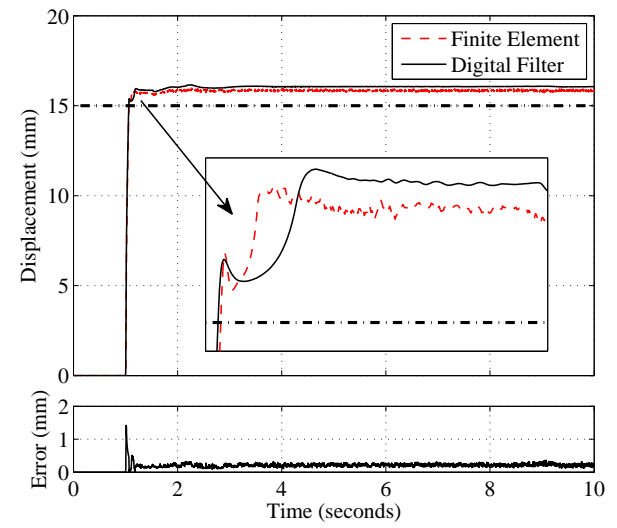

(a)

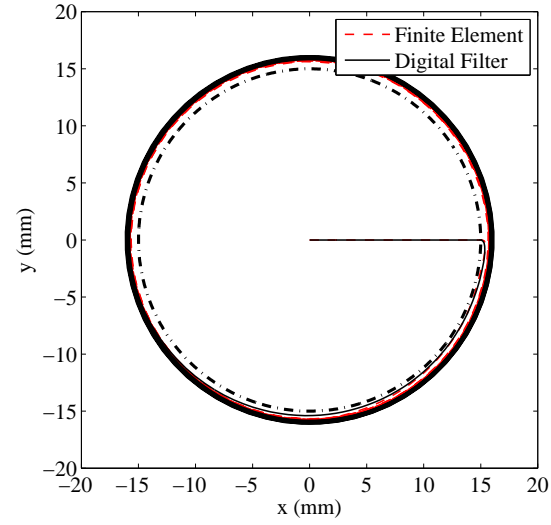

(b)

Fig. 12. Comparison of digital filter predictions with finite element predictions for the nonlinear transient response of the drillstring to an impulse of amplitude $I=3 I_{0}$ with coefficient of friction $\left(\mu_{0}=2\right)$ : (a) radial displacement as a function of time; and (b) $x-y$ trace of the displacement. The dashed line represents the borehole clearance radius. The drillstring makes contact with the borehole wall which then leads to sustained backward whirl. 


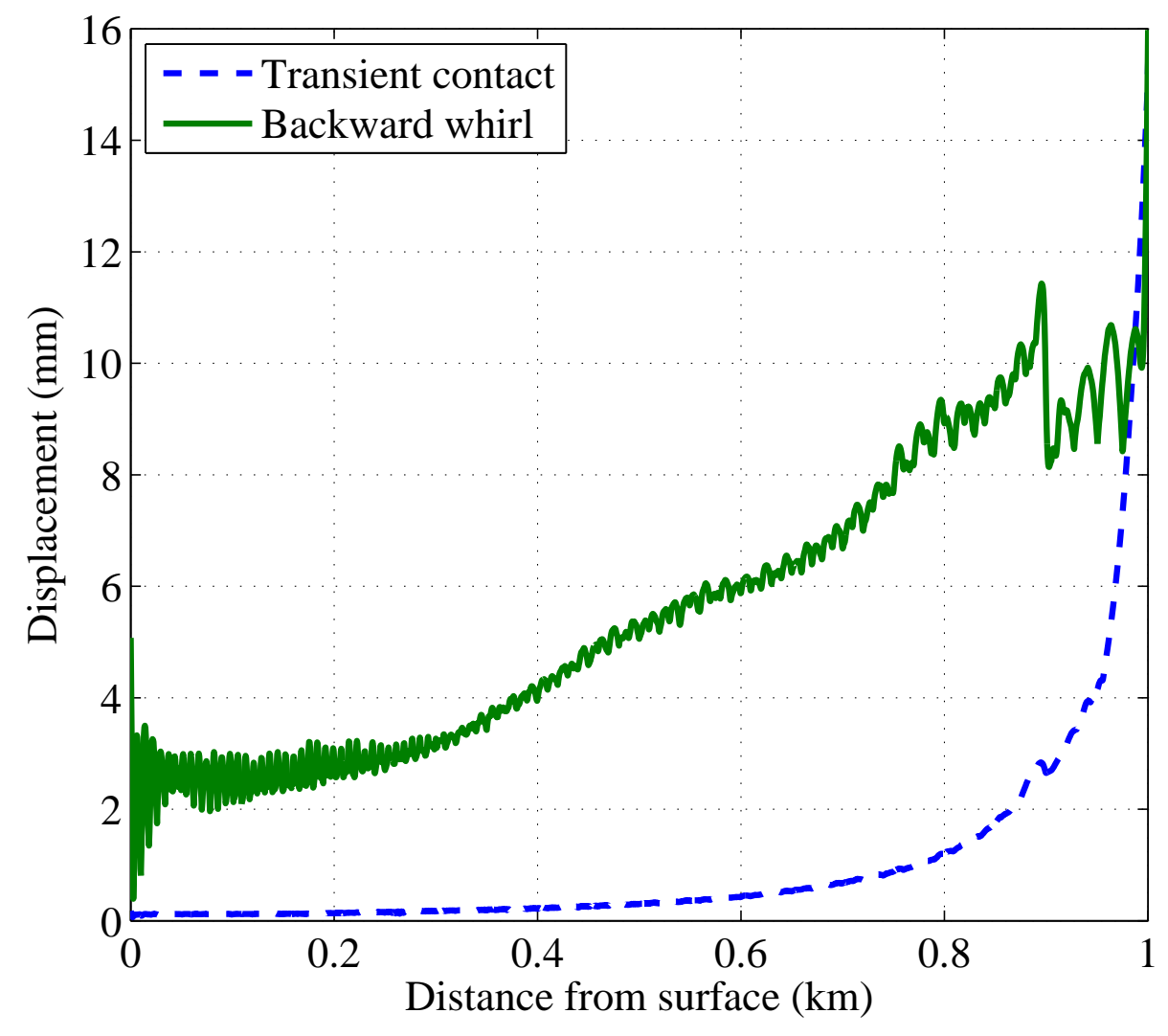

Fig. 13. Reconstruction of peak lateral amplitude of whole drillstring response. The dashed line shows the transient contact simulation with coefficient of friction $\mu_{0}=0.5$ and the solid line shows the example backward whirl simulation with coefficient of friction $\mu_{0}=2$. 


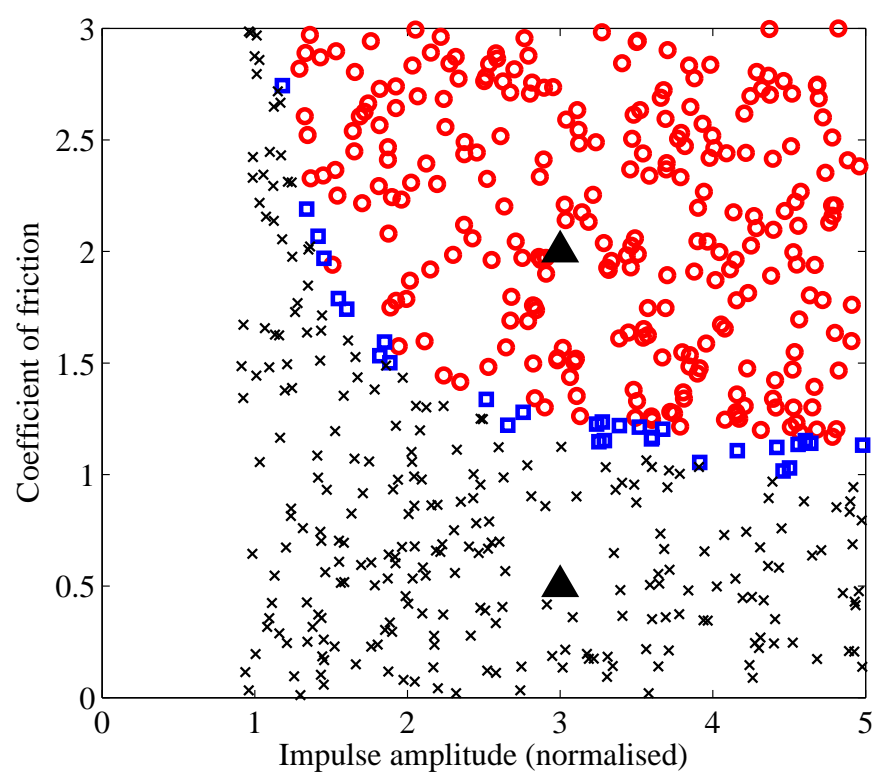

Fig. 14. Initiation conditions for backwards whirl, varying impulse amplitude $I / I_{0}$ and coefficient of friction $\mu_{0}$ : sustained backwards whirl (circles); transient response (crosses); and discrepancies between finite element and digital filter predictions (squares).

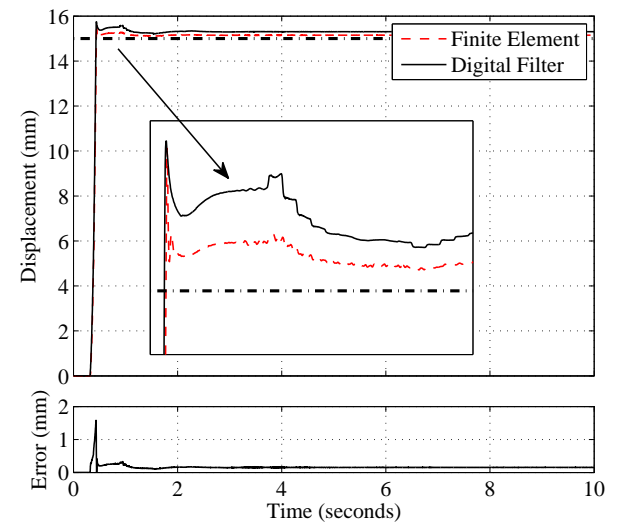

(a)

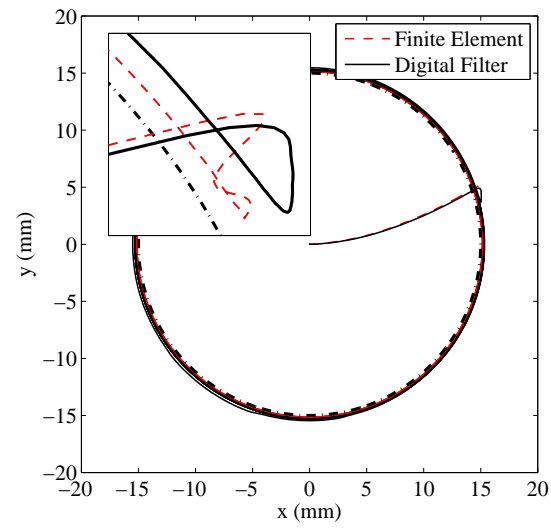

(b)

Fig. 15. Comparison of digital filter predictions with finite element predictions for the nonlinear transient response of the drillstring with an eccentricity of $m_{e} d=100 \mathrm{kgm}$ : (a) radial displacement as a function of time; and (b) $x-y$ trace of the displacement. The dashed line represents the borehole clearance radius. The drillstring makes contact with the borehole wall which then leads to sustained forwards whirl. 


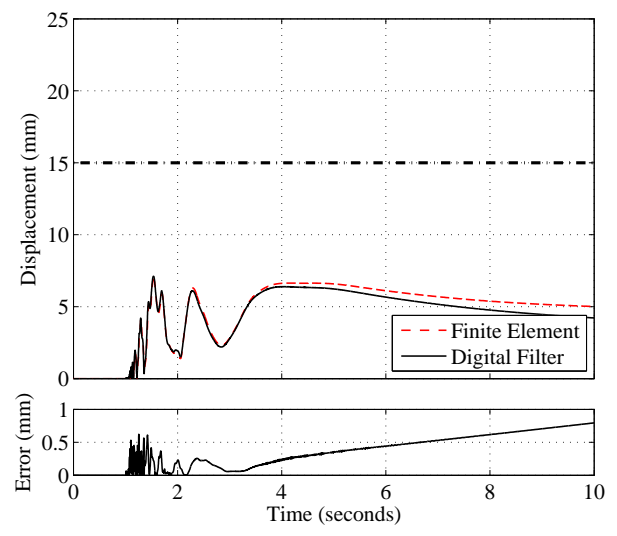

(a)

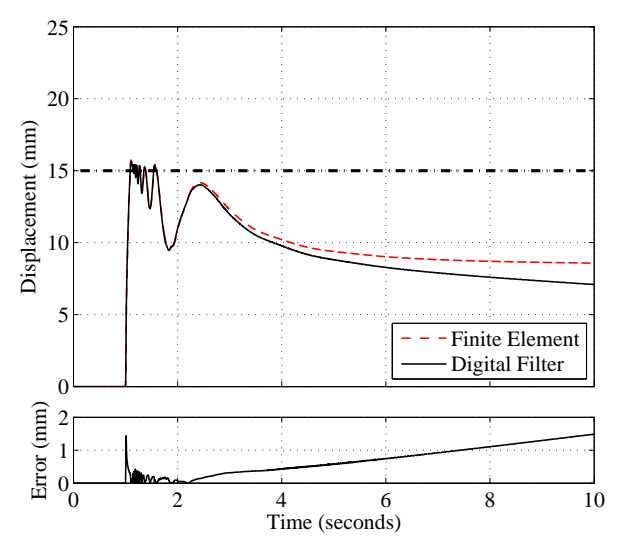

(c)

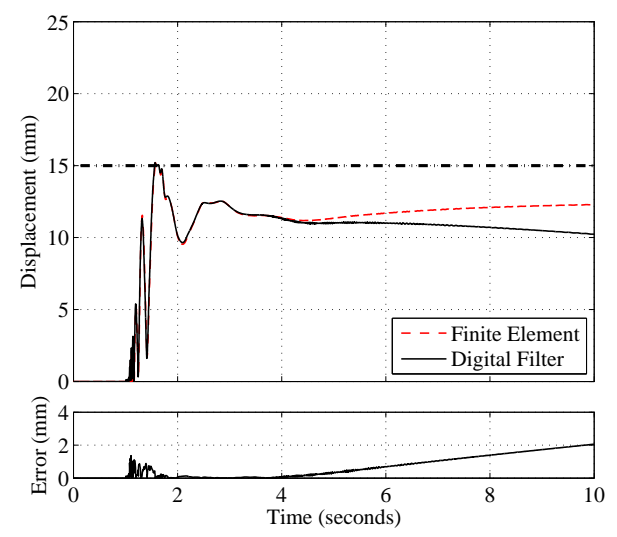

(e)

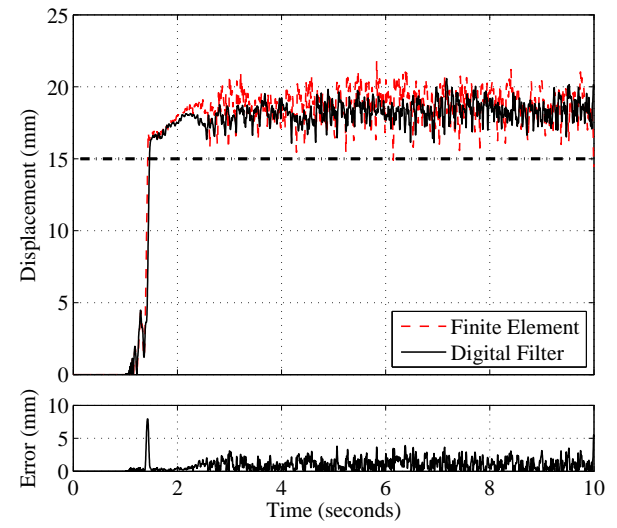

(b)

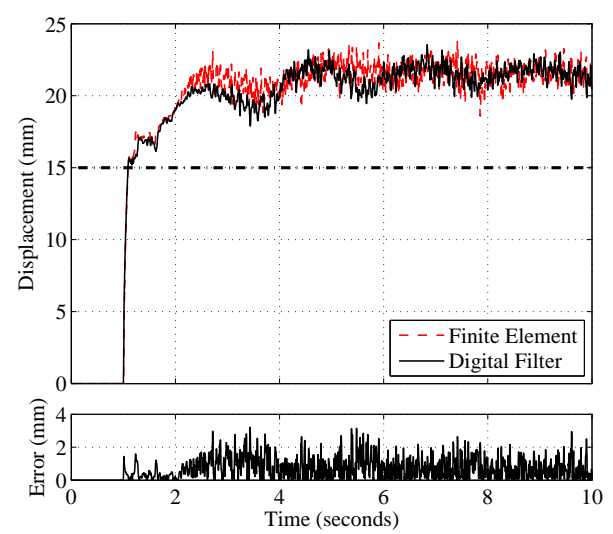

(d)

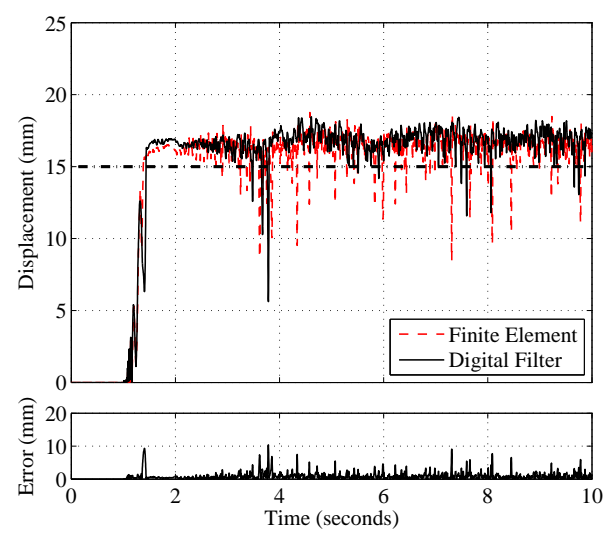

(f)

Fig. 16. Comparison of digital filter predictions with finite element predictions for the nonlinear transient response of the drillstring to an impulse of amplitude $I=3 I_{0}$. The left-side plots $(\mathrm{a}, \mathrm{c}, \mathrm{e})$ are for a coefficient of friction $\mu_{0}=0.5$; while the right-side plots $(\mathrm{b}, \mathrm{d}, \mathrm{f})$ are for $\mu_{0}=2$. The pair $(\mathrm{a}, \mathrm{b})$ show the predicted response for Site (1); $(\mathrm{c}, \mathrm{d})$ show Site $(2)$; and $(\mathrm{e}, \mathrm{f})$ show Site $(3)$. The horizontal dashed line shows the amplitude 


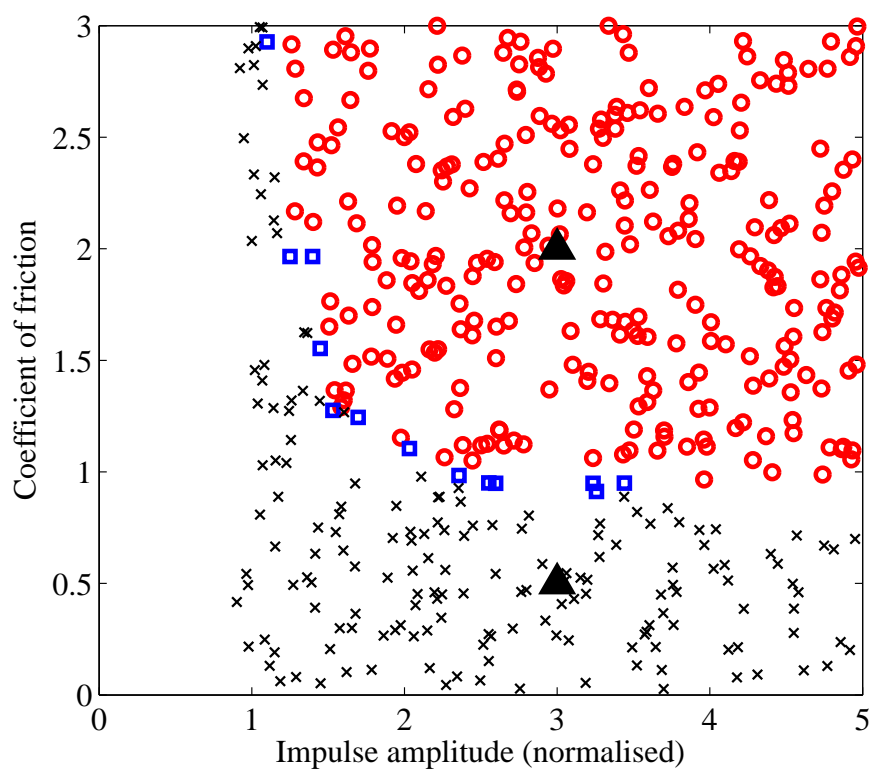

Fig. 17. Initiation conditions for backwards whirl for a drillstring with three points of contact, varying impulse amplitude $I / I_{0}$ and coefficient of friction $\mu_{0}$ : sustained backwards whirl (circles); transient response (crosses); and discrepancies between finite element and digital filter predictions (squares). 


\section{List of Tables}

2 Drillstring parameters used for test cases.

52 


\begin{tabular}{llll} 
Type & Property & Value \\
\hline \multirow{4}{*}{ General } & Shear modulus & $(G)$ & $80 \mathrm{GPa}$ \\
& Young's modulus & $(E)$ & $210 \mathrm{GPa}$ \\
& Density & $(\rho)$ & $7840 \mathrm{kgm}^{-3}$ \\
& Damping (torsion) & $\left(c_{t}\right)$ & $10 \mathrm{Nmsrad}^{-1}$ \\
& Damping (bending) & $\left(c_{b}\right)$ & $2 \mathrm{kNs}$ \\
\hline \multirow{4}{*}{ Drillpipe } & Number of segments & $(N)$ & 90 \\
& Lenternal radius of pipe & $\left(r_{1}\right)$ & $0.05 \mathrm{~m}$ \\
& External radius of pipe & $\left(R_{1}\right)$ & $0.125 \mathrm{~m}$ \\
& Internal radius of joint & $\left(r_{2}\right)$ & $0.05 \mathrm{~m}$ \\
& External radius of joint & $\left(R_{2}\right)$ & $0.15 \mathrm{~m}$ \\
& Length of joint & $\left(L_{2}\right)$ & $0.5 \mathrm{~m}$ \\
\hline \multirow{5}{*}{ Drillcollar } & Internal radius & $(N)$ & 10 \\
& External radius & $\left(r_{1}\right)$ & $0.05 \mathrm{~m}$ \\
& Length & $\left(R_{1}\right)$ & $0.15 \mathrm{~m}$ \\
& & $10 \mathrm{~m}$
\end{tabular}

Table 2

Drillstring parameters used for test cases. 